\title{
Quasigauge Spaces with Generalized Quasipseudodistances and Periodic Points of Dissipative Set-Valued Dynamic Systems
}

\author{
Kazimierz Włodarczyk and Robert Plebaniak \\ Department of Nonlinear Analysis, Faculty of Mathematics and Computer Science, \\ University of Łódz, Banacha 22, 90-238 Łódz, Poland \\ Correspondence should be addressed to Kazimierz Włodarczyk, wlkzxa@math.uni.lodz.pl
}

Received 13 September 2010; Revised 19 October 2010; Accepted 10 November 2010

Academic Editor: Jen Chih Yao

Copyright (c) 2011 K. Włodarczyk and R. Plebaniak. This is an open access article distributed under the Creative Commons Attribution License, which permits unrestricted use, distribution, and reproduction in any medium, provided the original work is properly cited.

\begin{abstract}
In quasigauge spaces, we introduce the families of generalized quasipseudodistances, and we define three kinds of dissipative set-valued dynamic systems with these families of generalized quasi-pseudodistances and with some families of not necessarily lower semicontinuous entropies and next, assuming that quasigauge spaces are left $K$ sequentially complete (but not necessarily Hausdorff), we prove that for each starting point each dynamic process or generalized sequence of iterations of these dissipative set-valued dynamic systems left converges and we also show that if an iterate of these dissipative set-valued dynamic systems is left quasiclosed, then these limit points are periodic points. Examples illustrating ideas, methods, definitions, and results are constructed.
\end{abstract}

\section{Introduction}

The study of quasigauge spaces, initiated by Reilly [1], has a long history. These spaces generalize topological spaces, quasiuniform spaces, and quasimetric spaces. Studies of asymmetric structures in these spaces and their applications to problems in theoretical computer science are important. There exists an extensive literature concerning unsymmetric distances, topological properties, and fixed point theory in these spaces. Some researches tools for many problems in these spaces were provided by Reilly [1, 2], Reilly et al. [3], Kelly [4], Subrahmanyam [5], Alemany and Romaguera [6], Romaguera [7], Stoltenberg [8], Wilson [9], Gregori and Romaguera [10], Lee et al. [11], Frigon [12], and Chiş-Novac et al. [13]. For quasiuniformities over the past 20 years, see also to the Fletcher and Lindgren book $[14]$ and to the Künzi surveys $[15,16]$.

Recall that a set-valued dynamic systems is defined as a pair $(X, T)$, where $X$ is a certain space and $T$ is a set-valued map $T: X \rightarrow 2^{X}$; in particular, a set-valued dynamic system 
includes the usual dynamic system, where $T$ is a single-valued map. Here, $2^{X}$ denotes the family of all nonempty subsets of a space $X$.

For each $x \in X$, a sequence $\left(w_{m}: m \in\{0\} \cup \mathbb{N}\right)$ such that

$$
\forall_{m \in\{0\} \cup \mathbb{N}}\left\{w_{m+1} \in T\left(w_{m}\right)\right\}, \quad w_{0}=x,
$$

is called a dynamic process or a trajectory starting at $w_{0}=x$ of the system $(X, T)$ (for details see Aubin and Siegel [17], Aubin and Ekeland [18], and Aubin and Frankowska [19]). For each $x \in X$, a sequence $\left(w_{m}: m \in\{0\} \cup \mathbb{N}\right)$, such that

$$
\forall_{m \in\{0\} \cup \mathbb{N}}\left\{w_{m+1} \in T^{[m+1]}(x)\right\}, \quad w_{0}=x,
$$

$T^{[m]}=T \circ T \circ \cdots \circ T$ ( $m$-times), $m \in \mathbb{N}$, is called a generalized sequence of iterations starting at $w_{0}=x$ of the system $(X, T)$ (for details see Yuan [20, page 557], Tarafdar and Vyborny [21], and Tarafdar and Yuan [22]). Each dynamic process starting from $w_{0}$ is a generalized sequence of iterations starting from $w_{0}$, but the converse may not be true; the set $T^{[\mathrm{m}]}\left(w_{0}\right)$ is, in general, bigger than $T\left(w_{m-1}\right)$. If $(X, T)$ is a single-valued, then, for each $x \in X$, a sequence $\left(w_{m}: m \in\{0\} \cup \mathbb{N}\right)$ such that

$$
\forall_{m \in\{0\} \cup \mathbb{N}}\left\{w_{m+1}=T^{[m+1]}(x)\right\}, \quad w_{0}=x,
$$

is called a Picard iteration starting at $w_{0}=x$ of the system $(X, T)$. If $(X, T)$ is a single valued, then (1.1)-(1.3) are identical.

If $(X, T)$ is a dynamic system, then by $\operatorname{Fix}(T), \operatorname{Per}(T)$, and $\operatorname{End}(T)$, we denote the sets of all fixed points, periodic points, and endpoints of $T$, respectively, that is, $\operatorname{Fix}(T)=\{w \in X: w \in$ $T(w)\}, \operatorname{Per}(T)=\left\{w \in X: w \in T^{[q]}(w)\right.$ for some $\left.q \in \mathbb{N}\right\}$, and $\operatorname{End}(T)=\{w \in X:\{w\}=T(w)\}$.

Let $X$ be a metric space with metric $d$, and let $(X, T)$ be a single-valued dynamic system. Racall that if $\exists_{\lambda \in[0,1)} \forall_{x, y \in X}\{d(T(x), T(y)) \leqslant \lambda d(x, y)\}$, then $(X, T)$ is called a Banach's contraction (Banach [23]). (X,T) is called contractive if $\forall_{x, y \in X}\{d(T(x), T(y))<d(x, y)\}$. If $\exists_{e>0} \forall_{x, y \in X}\{0<d(x, y)<\epsilon \Rightarrow d(T(x), T(y))<d(x, y)\}$, then $(X, T)$ is called $\epsilon$-contractive (Edelstein [24]). Contractive and $\epsilon$-contractive maps are some modifications of Banach's contractions. If

$$
\forall_{x \in X}\{d(x, T(x)) \leqslant \omega(x)-\omega(T(x))\}
$$

for some $\omega: X \rightarrow[0,+\infty)$, then $T$ is called Caristi's map (Caristi [25], Caristi and Kirk [26]) and $\omega$ is called entropy. Caristi's maps generalize Banach's contractions (for details see Kirk and Saliga [27, page 2766]). Recall that Ekeland's [28] variational principle concerning lower semicontinuous maps and Caristi's fixed point theorem (Caristi [25]) when entropy $\omega$ is lower semicontinuous are equivalent.

In metric spaces $(X, d)$, map $\omega: X \rightarrow[0,+\infty)$ is called a weak entropy or entropy of a set-valued dynamic system $(X, T)$ if

$$
\forall_{x \in X} \exists_{y \in T(x)}\{d(x, y) \leqslant \omega(x)-\omega(y)\}
$$


or

$$
\forall_{x \in X} \forall_{y \in T(x)}\{d(x, y) \leqslant \omega(x)-\omega(y)\}
$$

respectively, and $(X, T)$ is called weak dissipative or dissipative if it has a weak entropy or an entropy, respectively; here, $\omega$ is not necessarily lower semicontinuous. These two kinds of dissipative maps were introduced and studied by Aubin and Siegel [17]. If $(X, T)$ is a single valued, then (1.4)-(1.6) are identical.

Various results concerning the convergence of Picard iterations and the existence of periodic points, fixed points, and invariant sets for contractive and $\epsilon$-contractive singlevalued and set-valued dynamic systems in metric spaces have been established by Edelstein [24], Ding and Nadler Jr. [29], and Nadler Jr. [30]. Periodic point theorem for special singlevalued dynamic systems of Caristi's type in quasimetric spaces haS been obtained by Cirić [31, Theorem 2].

Investigations concerning the existence of fixed points and endpoints and convergence of dynamic processes or generalized sequences of iterations to fixed points or endpoints of single-valued and set-valued dissipative dynamic systems of the types (1.4)-(1.6) when entropy $\omega$ is not necessarily lower semicontinuous have been conducted by a number of authors in different settings; for example, see Aubin and Siegel [17], Kirk and Saliga [27], Yuan [20], Willems [32], Zangwill [33], Justman [34], Maschler and Peleg [35], and Petruşel and Sîntămărian [36].

In this paper, in quasigauge spaces (see Section 2), we introduce the families of generalized quasipseudodistances and define three new kinds of dissipative set-valued dynamic systems with these families of generalized quasipseudodistances and with some families of not necessarily lower semicontinuous entropies (see Section 3) and next, assuming that quasigauge spaces are left $K$ sequentially complete (but not necessarily Hausdorff), we prove that for each starting point each dynamic process or generalized sequence of iterations of these dissipative set-valued dynamic systems left converges, and we also show that if some iterates of these dissipative set-valued dynamic systems are left quasiclosed, then these limit points are periodic points (see Section 4). Examples are included (see Section 5).

The presented methods and results are different from those given in the literature and are new even for single-valued and set-valued dynamic systems in topological, quasiuniform, and quasimetric spaces.

This paper is a continuation of [37-41].

\section{Quasigauge Spaces}

The following terminologies will be much used.

Definition 2.1. Let $X$ be a nonempty set. A quasipseudometric on $X$ is a map $p: X \times X \rightarrow[0, \infty)$ such that

$$
\begin{aligned}
& \text { (D1) } \forall_{x \in X}\{p(x, x)=0\} \\
& \text { (D2) } \forall_{x, y, z \in X}\{p(x, z) \leqslant p(x, y)+p(y, z)\} . \\
& \text { If, additionally, } \\
& \text { (D3) } \forall_{x, y \in X}\{p(x, y)=0 \Rightarrow x=y\} \\
& \text { then } p \text { is called quasimetric on } X .
\end{aligned}
$$


Definition 2.2. Let $X$ be a nonempty set.

(i) Each family $D=\left\{p_{\alpha}: \alpha \in \mathscr{A}\right\}$ of quasipseudometrics $p_{\alpha}: X \times X \rightarrow[0, \infty), \alpha \in \mathcal{A}$ is called a quasigauge on $X$.

(ii) Let the family $D=\left\{p_{\alpha}: \alpha \in \mathscr{A}\right\}$ be a quasigauge on $X$. The topology $\tau(D)$ having as a subbase the family $B(D)=\left\{B\left(x, \varepsilon_{\alpha}\right): x \in X, \varepsilon_{\alpha}>0, \alpha \in \mathscr{A}\right\}$ of all balls $B\left(x, \varepsilon_{\alpha}\right)=$ $\left\{y \in X: p_{\alpha}(x, y)<\varepsilon_{\alpha}\right\}, x \in X, \varepsilon_{\alpha}>0, \alpha \in \mathcal{A}$ is called the topology induced by $D$ on $X$.

(iii) (Dugundji [42], Reilly $[1,2])$ A topological space $(X, \tau)$ such that there is a quasigauge $P$ on $X$ with $\tau=\tau(D)$ is called a quasigange space and is denoted by $(X, p)$.

Theorem 2.3 (see Reilly [1, Theorem 2.6]). Any topological space is a quasigauge space.

Definition 2.4. Let $X$ be a nonempty set.

(i) A quasiuniformity on $X$ is a filter $\mathcal{U}$ on $X \times X$ such that

(U1) $\forall U \in \mathcal{U}\{\Delta(X) \subseteq U\}$,

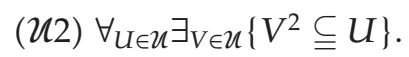

Here, $\Delta(X)=\{(x, x): x \in X\}$ denotes the diagonal of $X \times X$ and, for each $M \subset X \times X$, $M^{2}=\left\{(x, y) \in X \times X: \exists_{z \in X}\{(x, z) \in M \wedge(z, y) \in M\}\right\}$. The elements of $\mathcal{U}$ are called entourages (or vicinities).

(ii) A subfamily $\mathbb{B}$ of $\mathcal{U}$ is called a base of the quasiuniformity $\mathcal{U}$ on $X$ if $\forall U \in \mathcal{U} \exists_{V \in \mathcal{B}}\{V \subset$ $U\}$.

(iii) The topology $\tau(\mathcal{U})$ on $X$ induced by the quasiuniformity $\mathcal{U}$ on $X$ is $\{A \subseteq X$ : $\left.\forall_{x \in A} \exists_{U \in \mathcal{U}}\{U(x) \subseteq A\}\right\}$; here $U(x)=\{y \in X:(x, y) \in U\}$ whenever $U \in \mathcal{U}$ and $x \in X$. A neighborhood base for each point $x \in X$ is given by $\{U(x): U \in \mathcal{U}\}$.

(iv) If $\mathcal{U}$ is a quasiuniformity on $X$, then the pair $(X, \mathcal{U})$ is called a quasiuniform space.

Theorem 2.5 (see Reilly [1, Theorem 4.2]). Any quasiuniform space is a quasigauge space.

Definition 2.6. Let $(X, D)$ be a quasigauge space.

(i) (Reilly et al. [3, Definition $1(\mathrm{v})$ and page 129]) We say that a sequence $\left(w_{m}: m \in \mathbb{N}\right)$ in $X$ is left- $(D, K-)$ Cauchy sequence in $X$ if

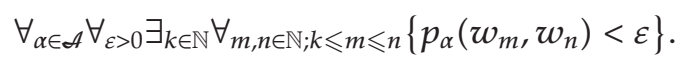

(ii) (Reilly et al. [3, Definition 1(i) and page 129]) We say that a sequence $\left(w_{m}: m \in \mathbb{N}\right)$ in $X$ is left $D$-Cauchy sequence in $X$ if

$$
\forall_{\alpha \in \mathscr{A}} \forall_{\varepsilon>0} \exists_{w \in X} \exists_{k \in \mathbb{N}} \forall_{m \in \mathbb{N} ; k \leqslant m}\left\{p_{\alpha}\left(w, w_{m}\right)<\varepsilon\right\} .
$$

(iii) We say that a sequence $\left(w_{m}: m \in \mathbb{N}\right)$ in $X$ is left convergent in $X$ if

$$
\exists_{w \in X} \forall_{\alpha \in \mathscr{A}} \forall_{\varepsilon>0} \exists_{k \in \mathbb{N}} \forall_{m \in \mathbb{N} ; k \leqslant m}\left\{p_{\alpha}\left(w, w_{m}\right)<\varepsilon\right\} .
$$


Fixed Point Theory and Applications

(iv) (Reilly [1, Definition 5.3] and [2, Definition 4]) If every left- ( $P, K-)$ Cauchy sequence in $X$ is left convergent to some point in $X$, then $(X, D)$ is called left $K$ sequentially complete quasigauge space.

(v) (Reilly [1, Definition 5.3] and [2, Definition 4]) If every left $p$-Cauchy sequence in $X$ is left convergent to some point in $X$, then $(X, P)$ is called left sequentially complete quasigauge space.

Remark 2.7. Let $(X, D)$ be a quasigauge space.

(a) (Reilly [2, page 131]) Every left- $(D, K$-) Cauchy sequence in $X$ is left $P$-Cauchy sequence in $X$.

(b) (Reilly [2, Example 1], Reilly et al. [3, Example 2], and Kelly [4, Example 5.8]) Every left convergent sequence in $X$ is left $P$-Cauchy sequence in $X$ and the converse is false.

(c) (Reilly et al. [3, Section 3]) Every left sequentially complete quasigauge space is left $K$ sequentially complete quasigauge space.

\section{Three Kinds of Dissipative Set-Valued Dynamic Systems in Quasigauge Spaces with Generalized Quasipseudodistances}

First, we introduce the concepts of $2 p$-family of generalized quasipseudodistances in quasigauge space $(X, p)$ and left- $(2 p, K-)$ Cauchy sequences in quasigauge space $(X, p)$ with $2 p$-family of generalized quasipseudodistances.

Definition 3.1. Let $(X, D)$ be a quasigauge space.

(i) The family $\mathcal{\partial}=\left\{J_{\alpha}: \alpha \in \mathcal{A}\right\}$ of maps $J_{\alpha}: X \times X \rightarrow[0, \infty), \alpha \in \mathcal{A}$, is said to be a $2 p$-family on $X$ if the following two conditions hold:

(21) $\forall_{\alpha \in \mathcal{A}} \forall_{x, y, z \in X}\left\{J_{\alpha}(x, z) \leqslant J_{\alpha}(x, y)+J_{\alpha}(y, z)\right\}$,

(22) for any sequence $\left(w_{m}: m \in \mathbb{N}\right)$ in $X$ such that

$$
\forall_{\alpha \in \mathscr{A}} \forall_{\varepsilon>0} \exists_{k \in \mathbb{N}} \forall_{m, n \in \mathbb{N} ; k \leqslant m \leqslant n}\left\{J_{\alpha}\left(w_{m}, w_{n}\right)<\varepsilon\right\},
$$

if there exists a sequence $\left(v_{m}: m \in \mathbb{N}\right)$ in $X$ satisfying

$$
\forall_{\alpha \in \mathscr{A}} \forall_{\varepsilon>0} \exists_{k \in \mathbb{N}} \forall_{m \in \mathbb{N} ; k \leqslant m}\left\{J_{\alpha}\left(w_{m}, v_{m}\right)<\varepsilon\right\},
$$

then

$$
\forall_{\alpha \in \mathcal{A}} \forall_{\varepsilon>0} \exists_{k \in \mathbb{N}} \forall_{m \in \mathbb{N} ; k \leqslant m}\left\{p_{\alpha}\left(w_{m}, v_{m}\right)<\varepsilon\right\} .
$$

(ii) The elements of $2 p$-family on $X$ are called generalized quasipseudodistances on $X$.

(iii) Let the family $\partial=\left\{J_{\alpha}: \alpha \in \mathcal{A}\right\}$ be a $\partial_{p}$-family on $X$. We say that a sequence $\left(w_{m}: m \in \mathbb{N}\right)$ in $X$ is left- $(2 p, K-)$ Cauchy sequence in $X$ if (3.1) holds. 
Remark 3.2. Let $X$ be a nonempty set.

(a) If $(X, p)$ is a quasigauge space, $\partial=\left\{J_{\alpha}: \alpha \in \mathcal{A}\right\}$ is a $2 p$-family on $X$ and $\forall_{\alpha \in \mathcal{A}} \forall_{x \in X}\left\{J_{\alpha}(x, x)=0\right\}$, then, for each $\alpha \in \mathcal{A}, J_{\alpha}$ is quasipseudometric.

(b) Each quasigauge $P$ on $X$ is $2 p$-family on $X$ and the converse is false (see Section 5 , e.g., in Example 5.1(II), if $x \notin E$, then $\left.\forall_{\alpha \in \mathcal{A}}\left\{J_{\alpha}(x, x)=c_{\alpha}>0\right\}\right)$.

Now, we introduce the following three kinds of dissipative set-valued dynamic systems in quasigauge spaces with generalized quasipseudodistances.

Definition 3.3. Let $(X, P)$ be a quasigauge space, and let $(X, T)$ be a set-valued dynamic system. Let $\partial=\left\{J_{\alpha}: \alpha \in \mathcal{A}\right\}, J_{\alpha}: X \times X \rightarrow[0, \infty), \alpha \in \mathcal{A}$ be a $2 p$-family on $X$, and let $\Gamma=\left\{\gamma_{\alpha}: \alpha \in \mathcal{A}\right\}, \gamma_{\alpha}: X \rightarrow[0, \infty), \alpha \in \mathcal{A}$ be a family of maps.

(i) We say that a sequence $\left(w_{m}: m \in\{0\} \cup \mathbb{N}\right)$ in $X$ is $(2, \Gamma)$ admissible if

$$
\forall_{\alpha \in \mathscr{A}} \forall_{m \in\{0\} \cup \mathbb{N}}\left\{J_{\alpha}\left(w_{m}, w_{m+1}\right) \leqslant \gamma_{\alpha}\left(w_{m}\right)-\gamma_{\alpha}\left(w_{m+1}\right)\right\}
$$

(ii) If the following two conditions hold:

(C1) $\emptyset \neq X_{0} \subset X$,

(C2) $x \in X_{0}$ if and only if there exists a $(2, \Gamma-)$ admissible dynamic process $\left(w_{m}\right.$ : $m \in\{0\} \cup \mathbb{N})$ starting at $w_{0}=x$ of the system $(X, T)$, then we say that $T$ is a weak $\left(2, \Gamma ; X_{0}\right)$ dissipative on $X$.

(iii) We say that $T$ is $(2, \Gamma)$-dissipative on $X$ if, for each $x \in X$, each dynamic process $\left(w_{m}: m \in\{0\} \cup \mathbb{N}\right)$ starting at $w_{0}=x$ of the system $(X, T)$ is $(2, \Gamma)$-admissible.

(iv) We say that $T$ is a strictly $(2, \Gamma)$ dissipative on $X$ if, for each $x \in X$, each generalized sequence of iterations $\left(w_{m}: m \in\{0\} \cup \mathbb{N}\right)$ starting at $w_{0}=x$ of the system $(X, T)$ is $(2, \Gamma)$ admissible.

If one from the conditions (ii)-(iv) holds, then we say that $(X, T)$ is a dissipative setvalued dynamic system with respect to $(2, \Gamma)$ (dissipative set-valued dynamic system, for short) and elements of the family $\Gamma$ we call entropies on $X$.

Remark 3.4. Let $(X, D)$ be a quasigauge space, and let $(X, T)$ be a set-valued dynamic system.

(a) If a sequence $\left(w_{m}: m \in\{0\} \cup \mathbb{N}\right)$ in $X$ is $(2, \Gamma)$ admissible, then, for each $k \in \mathbb{N}$, a sequence $\left(w_{m+k}: m \in\{0\} \cup \mathbb{N}\right)$ is $(2, \Gamma)$ admissible.

(b) By (a), if $T$ is a weak $\left(2, \Gamma ; X_{0}\right)$ dissipative on $X, x \in X_{0}$ and $\left(w_{m}: m \in\{0\} \cup \mathbb{N}\right)$ is a $(2, \Gamma)$-admissible dynamic process starting at $w_{0}=x$ of the system $(X, T)$, then $\forall_{m \in \mathbb{N}}\left\{w_{m} \in X_{0}\right\}$.

(c) If $(X, T)$ is a single-valued dynamic system, then (iii) and (iv) are identical. 
Proposition 3.5. Let $(X, P)$ be a quasigauge space, and let $(X, T)$ be a set-valued dynamic system.

(a) If $T$ is a weak $\left(2, \Gamma ; X_{0}\right)$ dissipative on $X$, then $\left(X_{0}, \mathbb{K}_{2 ; T}\right)$ is a set-valued dynamic system where, for each $x \in X_{0}$,

$$
\begin{aligned}
\mathbb{K}_{2 ; T}(x)= & \bigcup\left\{\left\{w_{0}, w_{1}, w_{2}, \ldots\right\}:\left(w_{m}: m \in\{0\} \cup \mathbb{N}\right) \in \mathcal{K}_{2}(T, x)\right\} \\
\mathcal{K}_{2}(T, x)= & \left\{\left(w_{m}: m \in\{0\} \cup \mathbb{N}\right): w_{0}=x\right. \\
& \left.\wedge \forall_{m \in\{0\} \cup \mathbb{N}}\left\{w_{m+1} \in T\left(w_{m}\right) \wedge \forall_{\alpha \in \mathcal{A}}\left\{J_{\alpha}\left(w_{m}, w_{m+1}\right) \leqslant \gamma_{\alpha}\left(w_{m}\right)-\gamma_{\alpha}\left(w_{m+1}\right)\right\}\right\}\right\} .
\end{aligned}
$$

(b) If $T$ is $(2, \Gamma)$ dissipative on $X$, then $\left(X, \mathbb{W}_{2 ; T}\right)$ is a set-valued dynamic system where, for each $x \in X$,

$$
\begin{gathered}
\mathbb{W}_{2 ; T}(x)=\bigcup\left\{\left\{w_{0}, w_{1}, w_{2}, \ldots\right\}:\left(w_{m}: m \in\{0\} \cup \mathbb{N}\right) \in \mathcal{W}_{2}(T, x)\right\}, \\
\mathcal{W}_{2}(T, x)=\left\{\left(w_{m}: m \in\{0\} \cup \mathbb{N}\right): w_{0}=x \wedge \forall_{m \in\{0\} \cup \mathbb{N}}\left\{w_{m+1} \in T\left(w_{m}\right)\right\}\right\} .
\end{gathered}
$$

(c) If $T$ is a strictly $(2, \Gamma)$ dissipative on $X$, then $\left(X, \mathbb{S}_{2 ; T}\right)$ is a set-valued dynamic system where, for each $x \in X$,

$$
\begin{gathered}
\mathbb{S}_{2 ; T}(x)=\bigcup\left\{\left\{w_{0}, w_{1}, w_{2}, \ldots\right\}:\left(w_{m}: m \in\{0\} \cup \mathbb{N}\right) \in \mathcal{S}_{2}(T, x)\right\} \\
\mathcal{S}_{2}(T, x)=\left\{\left(w_{m}: m \in\{0\} \cup \mathbb{N}\right): w_{0}=x \wedge \forall_{m \in\{0\} \cup \mathbb{N}}\left\{w_{m+1} \in T^{[m+1]}\left(w_{0}\right)\right\}\right\} .
\end{gathered}
$$

Proof. The fact that $\mathbb{K}_{2 ; T}: X_{0} \rightarrow 2^{X_{0}}, \mathbb{W}_{2 ; T}: X \rightarrow 2^{X}$, and $\mathbb{S}_{2 ; T}: X \rightarrow 2^{X}$ follows from (1.1), (1.2), Definition 3.3, Remark 3.4, and (3.5)-(3.10).

Remark 3.6. By Definition 3.3 and Proposition 3.5, we obtain the following.

(a) If $T$ is $(2, \Gamma)$ dissipative on $X$, then $T$ is a weak $\left(2, \Gamma ; X_{0}\right)$ dissipative on $X$ for $X_{0}=X$ and $\forall_{x \in X_{0}}\left\{\mathbb{K}_{2 ; T}(x)=\mathbb{W}_{2 ; T}(x)\right\}$.

(b) If $T$ is strictly $(2, \Gamma)$ dissipative on $X$, then $T$ is $(2, \Gamma)$ dissipative on $X$ and $\forall_{x \in X}\left\{\mathbb{W}_{2 ; T}(x) \subset \mathbb{S}_{2 ; T}(x)\right\}$.

\section{Convergence of Dynamic Processes and Generalized Sequences of Iterations and Periodic Points of Dissipative Set-Valued Dynamic Systems in Quasigauge Spaces with Generalized Quasipseudodistances}

We first recall the definition of closed maps in topological spaces given in Berge [43] and Klein and Thompson [44]. 
Definition 4.1. Let $L$ be a topological vector space. The set-valued dynamic system $(X, T)$ is called closed if whenever $\left(w_{m}: m \in \mathbb{N}\right)$ is a sequence in $X$ converging to $w \in X$ and $\left(v_{m}: m \in \mathbb{N}\right)$ is a sequence in $X$ satisfying the condition $\forall_{m \in \mathbb{N}}\left\{v_{m} \in T\left(w_{m}\right)\right\}$ and converging to $v \in X$, then $v \in T(w)$.

By Definition 2.6(iii), we are able to revise the above definition, and we define left quasiclosed maps and left quasiclosed sets in quasigauge spaces as follows.

Definition 4.2. Let $(X, D)$ be a left $K$ sequentially complete quasigauge space.

(i) The set-valued dynamic system $(X, T)$ is called left quasiclosed if whenever $\left(w_{m}\right.$ : $m \in \mathbb{N}$ ) is a sequence in $X$ left converging to each point of the set $W \subset X$ and $\left(v_{m}: m \in \mathbb{N}\right)$ is a sequence in $X$ satisfying the condition $\forall_{m \in \mathbb{N}}\left\{v_{m} \in T\left(w_{m}\right)\right\}$ and left converging to each point of the set $V \subset X$, then $\exists_{v \in V} \forall_{w \in W}\{v \in T(w)\}$.

(ii) For an arbitrary subset $E$ of $X$, the left quasi-closure of $E$, denoted by $\mathrm{cl}_{\mathrm{L}}(E)$, is defined as the set

$$
\operatorname{cl}_{\mathrm{L}}(E)=\left\{w \in X: \exists_{\left(w_{m}: m \in \mathbb{N}\right) \subset E} \forall_{\alpha \in \mathcal{A}} \forall_{\varepsilon>0} \exists_{k \in \mathbb{N}} \forall_{m \in \mathbb{N} ; k \leqslant m}\left\{p_{\alpha}\left(w, w_{m}\right)<\varepsilon\right\}\right\} .
$$

(iii) The subset $E$ of $X$ is said to be left quasiclosed subset in $X$ if $\operatorname{cl}_{\mathrm{L}}(E)=E$.

Remark 4.3. Let $(X, D)$ be a left $K$ sequentially complete quasigauge space. For each subset $E$ of $X, E \subset \mathrm{cl}_{\mathrm{L}}(E)$. Indeed, by Definition 4.2(ii) and (D1), for each $w \in E$, the sequence $\left(w_{m}: m \in \mathbb{N}\right)$, where $\forall_{m \in \mathbb{N}}\left\{w_{m}=w\right\}$, is left convergent to $w$.

Now we are ready to prove the following main result of this paper.

Theorem 4.4. Let $(X, D)$ be a left $K$ sequentially complete quasigange space, and let $(X, T)$ be a setvalued dynamic system. Let $\partial=\left\{J_{\alpha}: \alpha \in \mathcal{A}\right\}, J_{\alpha}: X \times X \rightarrow[0, \infty), \alpha \in \mathcal{A}$ be a $\partial_{p}$-family on $X$ and let $\Gamma=\left\{\gamma_{\alpha}: \alpha \in \mathcal{A}\right\}, \gamma_{\alpha}: X \rightarrow[0, \infty), \alpha \in \mathcal{A}$ be a family of maps. The following hold.

(A) $\left(A_{1}\right)$ If $T$ is weak $\left(2, \Gamma ; X_{0}\right)$ dissipative on $X$, then, for each $x \in X_{0}$ and for each dynamic process $\left(w_{m}: m \in\{0\} \cup \mathbb{N}\right) \in \mathcal{K}_{2}(T, x)$, there exists a nonempty set $W \subset c_{L}\left(X_{0}\right)$ such that, for each $w \in W,\left(w_{m}: m \in\{0\} \cup \mathbb{N}\right)$ is left convergent to $w$.

$\left(A_{2}\right)$ If , in addition, the map $T^{[q]}$ is left quasiclosed in $X$ for some $q \in \mathbb{N}$, then there exists $w \in W$ such that $w \in T^{[q]}(w)$.

(B) $\left(B_{1}\right)$ If $T$ is $(2, \Gamma)$ dissipative on $X$, then, for each $x \in X$ and for each dynamic process $\left(w_{m}: m \in\{0\} \cup \mathbb{N}\right) \in \mathcal{W}_{2}(T, x)$, there exists a nonempty set $W \subset X$ such that, for each $w \in W,\left(w_{m}: m \in\{0\} \cup \mathbb{N}\right)$ is left convergent to $w$.

$\left(B_{2}\right)$ If, in addition, the map $T^{[q]}$ is left quasiclosed in $X$ for some $q \in \mathbb{N}$, then there exists $w \in W$ such that $w \in T^{[q]}(w)$.

(C) $\left(C_{1}\right)$ If $T$ is strictly $(2, \Gamma)$ dissipative on $X$, then, for each $x \in X$ and for each generalized sequence of iterations $\left(w_{m}: m \in\{0\} \cup \mathbb{N}\right) \in \mathcal{S}_{2}(T, x)$, there exists a nonempty set $W \subset X$ such that, for each $w \in W,\left(w_{m}: m \in\{0\} \cup \mathbb{N}\right)$ is left convergent to $w$.

$\left(C_{2}\right)$ If, in addition, the map $T^{[q]}$ is left quasiclosed in $X$ for some $q \in \mathbb{N}$, then, for each $x \in X$, there exists a generalized sequence of iterations $\left(w_{m}: m \in\{0\} \cup \mathbb{N}\right) \in \mathcal{S}_{2}(T, x), a$ nonempty set $W \subset X$ and $w \in W$ such that $\left(w_{m}: m \in\{0\} \cup \mathbb{N}\right)$ is left convergent to each points of $W$ and $w \in T^{[q]}(w)$. 
Proof. The proof will be broken into five steps.

Step 1. Let (i) $x \in X_{0}$ and $\left(w_{m}: m \in\{0\} \cup \mathbb{N}\right) \in \mathcal{K}_{2}(T, x)$ or (ii) $x \in X$ and $\left(w_{m}: m \in\right.$ $\{0\} \cup \mathbb{N}) \in \mathcal{W}_{2}(T, x) \cup \mathcal{S}_{2}(T, x)$. We show that $\left(w_{m}: m \in\{0\} \cup \mathbb{N}\right)$ is left- $(2 p, K-)$ Cauchy sequence in $X$, that is,

$$
\forall_{\alpha \in \mathscr{A}} \forall_{\varepsilon>0} \exists_{k \in \mathbb{N}} \forall_{m, n \in \mathbb{N} ; k \leqslant m \leqslant n}\left\{J_{\alpha}\left(w_{m}, w_{n}\right)<\varepsilon\right\} .
$$

Indeed, by (3.6), (3.8), (3.10), Definition 3.3(iii) and (iv), and definition of 2 , $\forall_{\alpha \in \mathscr{A}} \forall_{m \in\{0\} \cup \mathbb{N}}\left\{\gamma_{\alpha}\left(w_{m+1}\right) \leqslant \gamma_{\alpha}\left(w_{m}\right)\right\}$. According to the fact that $\forall_{\alpha \in \mathcal{A}} \forall_{x \in X}\left\{\gamma_{\alpha}(x) \geqslant 0\right\}$, we conclude that, for each $\alpha \in \mathcal{A}$, the sequence $\left(\gamma_{\alpha}\left(w_{m}\right): m \in\{0\} \cup \mathbb{N}\right)$ is bounded from below and nonincreasing. Hence, we have

$$
\forall_{\alpha \in \mathcal{A}} \exists_{u_{\alpha} \geqslant 0}\left\{\lim _{m \rightarrow \infty}\left|\gamma_{\alpha}\left(w_{m}\right)-u_{\alpha}\right|=0\right\} .
$$

Let now $\alpha_{0} \in \mathcal{A}$ and $\varepsilon_{0}>0$ be arbitrary and fixed. By (4.3),

$$
\exists_{n_{0} \in \mathbb{N}} \forall_{m ; n_{0} \leqslant m}\left\{\left|\gamma_{\alpha_{0}}\left(w_{m}\right)-u_{\alpha_{0}}\right|<\frac{\varepsilon_{0}}{2}\right\} .
$$

Furthermore, for $n_{0} \leqslant m \leqslant n$, using (21) and (3.4), we obtain $0 \leqslant J_{\alpha_{0}}\left(w_{m}, w_{n}\right) \leqslant$ $\sum_{k=m}^{n-1} J_{\alpha_{0}}\left(w_{k}, w_{k+1}\right) \leqslant \gamma_{\alpha_{0}}\left(w_{m}\right)-\gamma_{\alpha_{0}}\left(w_{n}\right)$ and next, by (4.4), we have that $J_{\alpha_{0}}\left(w_{m}, w_{n}\right) \leqslant$ $\gamma_{\alpha_{0}}\left(w_{m}\right)-\gamma_{\alpha_{0}}\left(w_{n}\right)=\left|\gamma_{\alpha_{0}}\left(w_{m}\right)-u_{\alpha_{0}}-\gamma_{\alpha_{0}}\left(w_{n}\right)+u_{\alpha_{0}}\right| \leqslant\left|\gamma_{\alpha_{0}}\left(w_{m}\right)-u_{\alpha_{0}}\right|+\left|\gamma_{\alpha_{0}}\left(w_{n}\right)-u_{\alpha_{0}}\right|<$ $\varepsilon_{0} / 2+\varepsilon_{0} / 2=\varepsilon_{0}$. Therefore, (4.2) holds.

Step 2. Let (i) $x \in X_{0}$ and $\left(w_{m}: m \in\{0\} \cup \mathbb{N}\right) \in \mathcal{K}_{2}(T, x)$ or(ii) $x \in X$ and ( $w_{m}: m \in$ $\{0\} \cup \mathbb{N}) \in \mathcal{W}_{2}(T, x) \cup \mathcal{S}_{2}(T, x)$. We show that $\left(w_{m}: m \in\{0\} \cup \mathbb{N}\right)$ is left- $\left(D, K_{-}\right)$Cauchy sequence in $X$, that is,

$$
\forall_{\alpha \in \mathcal{A}} \forall_{\varepsilon>0} \exists_{k \in \mathbb{N}} \forall_{m, n \in \mathbb{N} ; k \leqslant m \leqslant n}\left\{p_{\alpha}\left(w_{m}, w_{n}\right)<\varepsilon\right\} .
$$

Indeed, by (4.2), $\forall_{\alpha \in \mathscr{A}} \forall_{\varepsilon>0} \exists_{k \in \mathbb{N}} \forall_{m \geqslant k} \forall_{l \in\{0\} \cup \mathbb{N}}\left\{J_{\alpha}\left(w_{m}, w_{l+m}\right)<\varepsilon\right\}$. Hence, if $i_{0} \in\{0\} \cup \mathbb{N}$ is arbitrary and fixed and if we define a sequence $\left(v_{m}: m \in \mathbb{N}\right)$ as $v_{m}=w_{i_{0}+m}$ for $m \in \mathbb{N}$, then we obtain

$$
\forall_{\alpha \in \mathcal{A}} \forall_{\varepsilon>0} \exists_{k \in \mathbb{N}} \forall_{m \geqslant k}\left\{J_{\alpha}\left(w_{m}, v_{m}\right)<\varepsilon\right\}
$$

By (22), (4.2), and (4.6),

$$
\forall_{\alpha \in \mathcal{A}} \forall_{\varepsilon>0} \exists_{k \in \mathbb{N}} \forall_{m \geqslant k}\left\{p_{\alpha}\left(w_{m}, v_{m}\right)<\varepsilon\right\}
$$

Consequence of (4.7) and the definition of $\left(v_{m}: m \in \mathbb{N}\right)$ is

$$
\forall_{\alpha \in \mathcal{A}} \forall_{\varepsilon>0} \exists_{k \in \mathbb{N}} \forall_{m \geqslant k}\left\{p_{\alpha}\left(w_{m}, w_{i_{0}+m}\right)<\varepsilon\right\}
$$


Now, let $\alpha_{0} \in \mathcal{A}, \varepsilon_{0}>0$ be arbitrary and fixed. By (4.2),

$$
\exists_{n_{1} \in \mathbb{N}} \forall_{m \geqslant n_{1}} \forall_{l \in\{0\} \cup \mathbb{N}}\left\{J_{\alpha_{0}}\left(w_{m}, w_{l+m}\right)<\varepsilon_{0}\right\} .
$$

From (4.8), we get

$$
\exists_{n_{2} \in \mathbb{N}} \forall_{m \geqslant n_{2}} \forall_{i \in\{0\} \cup \mathbb{N}}\left\{p_{\alpha_{0}}\left(w_{m}, w_{i+m}\right)<\varepsilon_{0}\right\}
$$

Let $n_{0}=\max \left\{n_{1}, n_{2}\right\}+1$. Hence, if $n_{0} \leqslant m \leqslant n$, then $n=i_{0}+m$ for some $i_{0} \in\{0\} \cup \mathbb{N}$. Therefore, by $(4.9)$ and $(4.10), p_{\alpha_{0}}\left(w_{m}, w_{n}\right)=p_{\alpha_{0}}\left(w_{m}, w_{i_{0}+m}\right)<\varepsilon_{0}$. The proof of (4.5) is complete.

Step 3. Let (i) $x \in X_{0}$ and $\left(w_{m}: m \in\{0\} \cup \mathbb{N}\right) \in \mathcal{K}_{2}(T, x)$ or (ii) $x \in X$ and ( $w_{m}: m \in$ $\{0\} \cup \mathbb{N}) \in \mathcal{W}_{2}(T, x) \cup \mathcal{S}_{2}(T, x)$. We show that $\left(w_{m}: m \in\{0\} \cup \mathbb{N}\right)$ is left $D$-Cauchy sequence in $X$, that is,

$$
\forall_{\alpha \in \mathcal{A}} \forall_{\varepsilon>0} \exists_{w \in X} \exists_{k \in \mathbb{N}} \forall_{m \in \mathbb{N} ; k \leqslant m}\left\{p_{\alpha}\left(w, w_{m}\right)<\varepsilon\right\} .
$$

Indeed, by Remark 2.7(a), property (4.11) is a consequence of Step 2.

Step 4. Assertions of (A) and (B) hold.

Indeed, let (i) $x \in X_{0}$ and $\left(w_{m}: m \in\{0\} \cup \mathbb{N}\right) \in \mathcal{K}_{2}(T, x)$ or (ii) $x \in X$ and $\left(w_{m}: m \in\right.$ $\{0\} \cup \mathbb{N}) \in \mathcal{W}_{2}(T, x)$.

Since $\forall_{m \in\{0\} \cup \mathbb{N}}\left\{w_{m} \in \mathbb{K}_{2 ; T}(x)\right\}$ or $\forall_{m \in\{0\} \cup \mathbb{N}}\left\{w_{m} \in \mathbb{W}_{2 ; T}(x)\right\}, X$ is left $K$ sequentially complete quasigauge space and (4.5) holds; therefore, by Definition 2.6(iv), we claim that there exists a nonempty set $W \subset \mathrm{cl}_{\mathrm{L}}\left(\mathbb{K}_{2 ; T}(x)\right)$ or $W \subset \mathrm{cl}_{\mathrm{L}}\left(\mathbb{W}_{2 ; T}(x)\right)$, respectively, where $\mathbb{K}_{2 ; T}(x) \subset X_{0}, \operatorname{cl}_{\mathrm{L}}\left(\mathbb{K}_{2 ; T}(x)\right) \subset \mathrm{cl}_{\mathrm{L}}\left(X_{0}\right), \mathbb{W}_{2 ; T}(x) \subset X$, and $\mathrm{cl}_{\mathrm{L}}(X)=X$, such that the sequence $\left(w_{m}: m \in\{0\} \cup \mathbb{N}\right)$ is left convergent to each point $w$ of $W$.

Now, we see that if $T^{[q]}$ is left quasiclosed for some $q \in \mathbb{N}$, then there exists a point $w \in W$ such that $w \in T^{[q]}(w)$. Indeed, by (1.1), we conclude that

$$
\forall_{m \in \mathbb{N}}\left\{w_{m} \in T\left(w_{m-1}\right) \subset T^{[2]}\left(w_{m-2}\right) \subset \cdots \subset T^{[m-1]}\left(w_{1}\right) \subset T^{[m]}\left(w_{0}\right)\right\}
$$

which gives

$$
w_{m q+k} \in T^{[q]}\left(w_{(m-1) q+k}\right) \quad \text { for } k=1,2, \ldots, q, m \in \mathbb{N}
$$

It is clear that, for each $k=1,2, \ldots, q$, the sequences $\left(w_{m q+k}: m \in\{0\} \cup \mathbb{N}\right)$ and $\left(w_{(m-1) q+k}\right.$ : $m \in\{0\} \cup \mathbb{N})$, as subsequences of $\left(w_{m}: m \in\{0\} \cup \mathbb{N}\right)$, also left converge to each point of $W$. Further, since $T^{[q]}$ is left quasiclosed in $X$, by (4.13) and Definition 4.2(i), we obtain $\exists_{v \in V=W} \forall_{w \in W}\left\{v \in T^{[q]}(w)\right\}$, which gives $\exists_{w \in W}\left\{w \in T^{[q]}(w)\right\}$.

Step 5. Assertion of $\left(\mathrm{C}_{1}\right)$ holds.

Indeed, let $x \in X$ and $\left(w_{m}: m \in\{0\} \cup \mathbb{N}\right) \in \mathcal{S}_{2}(T, x)$ be arbitrary and fixed. By Step 2 and Proposition 3.5(c), we claim that $\forall_{m \in\{0\} \cup \mathbb{N}}\left\{w_{m} \in T^{[m]}(x) \subset \mathbb{S}_{2 ; T}(x) \subset X\right\},\left(w_{m}\right.$ : 
$m \in\{0\} \cup \mathbb{N})$ is left $(D, K)$-Cauchy sequence in left $K$-sequentially complete quasigauge space $X$, and, by Definition 2.6(iv), there exists a nonempty set $W \subset \mathrm{cl}_{\mathrm{L}}\left(\mathbb{S}_{2 ; T}(x)\right)$ such that the sequence $\left(w_{m}: m \in\{0\} \cup \mathbb{N}\right)$ is left convergent to each point $w$ of $W$. This gives that assertion of $\left(\mathrm{C}_{1}\right)$ holds.

Step 6. Assertion of $\left(\mathrm{C}_{2}\right)$ holds.

Initially, we will prove that if, for some $q \in \mathbb{N}, T^{[q]}$ is left quasiclosed in $X$, then, for each $x \in X$, we may construct a generalized sequence of iterations $\left(w_{m}: m \in\{0\} \cup \mathbb{N}\right) \in \mathcal{S}_{2}(T, x)$ which converge to each point $w$ of some nonempty set $W \subset \mathrm{cl}_{\mathrm{L}}\left(\mathbb{S}_{2 ; T}(x)\right)$, and the following property holds: $\exists_{w \in W}\left\{w \in T^{[q]}(w)\right\}$.

Indeed, let $x \in X$ and $k \in\{1,2, \ldots, q\}$ be arbitrary and fixed. First, we construct a sequence $\left(u_{m q+k}: m \in\{0\} \cup \mathbb{N}\right)$ as follows. For $m=0$, we define $u_{k}$ as an arbitrary and fixed point satisfying $u_{k} \in T^{[k]}\left(w_{0}\right)$. Then, we have that $T^{[q]}\left(u_{k}\right) \subset T^{[q+k]}\left(w_{0}\right)$ and, for $m=1$, we define $u_{q+k}$ as an arbitrary and fixed point satisfying $u_{q+k} \in T^{[q]}\left(u_{k}\right) \subset T^{[q+k]}\left(w_{0}\right)$. Then, we have that $T^{[q]}\left(u_{q+k}\right) \subset T^{[2 q+k]}\left(w_{0}\right)$ and, for $m=2$, we define $u_{2 q+k}$ as an arbitrary and fixed point satisfying $u_{2 q+k} \in T^{[q]}\left(u_{q+k}\right) \subset T^{[2 q+k]}\left(w_{0}\right)$. In general, for each $m \in\{0\} \cup \mathbb{N}$, if we define $u_{(m-1) q+k}$ satisfying $u_{(m-1) q+k} \in T^{[q]}\left(u_{(m-2) q+k}\right) \subset T^{[q(m-1)+k]}\left(w_{0}\right)$, then we have that $T^{[q]}\left(u_{(m-1) q+k}\right) \subset T^{[m q+k]}\left(w_{0}\right)$ and define $u_{m q+k}$ as an arbitrary and fixed point satisfying $u_{m q+k} \in T^{[q]}\left(u_{(m-1) q+k}\right) \subset T^{[m q+k]}\left(w_{0}\right)$.

Consequently, for arbitrary and fixed $x \in X$ and $k \in\{1,2, \ldots, q\}$, there exists a sequence $\left(u_{m q+k}: m \in\{0\} \cup \mathbb{N}\right)$ satisfying

$$
\begin{gathered}
u_{k} \in T^{[k]}\left(w_{0}\right), \\
u_{m q+k} \in T^{[q]}\left(u_{(m-1) q+k}\right) \subset T^{[m q+k]}\left(w_{0}\right), \quad m \in \mathbb{N} .
\end{gathered}
$$

Let now $\left(w_{m}: m \in\{0\} \cup \mathbb{N}\right)$ be an arbitrary and fixed sequence satisfying (1.2) and the condition

$$
w_{m q+k}=u_{m q+k}, \quad m \in\{0\} \cup \mathbb{N} .
$$

Obviously, by Step 5, $\left(w_{m}: m \in\{0\} \cup \mathbb{N}\right)$ is left convergent to each point $w$ of some set $W \subset \mathrm{cl}_{\mathrm{L}}\left(\mathbb{S}_{2 ; T}(x)\right)$. Moreover, $\left(u_{m q+k}: m \in\{0\} \cup \mathbb{N}\right)$ and $\left(u_{(m-1) q+k}: m \in \mathbb{N}\right)$, as subsequences of $\left(w_{m}: m \in\{0\} \cup \mathbb{N}\right)$, also converge to each point $w$ of some set $W$. Hence, using (4.15), (4.16), assumption in $\left(C_{2}\right)$, and Definition 4.2(i), we get that $\exists_{v \in V=W} \forall_{w \in W}\left\{v \in T^{[q]}(w)\right\}$, which gives $\exists_{w \in W}\left\{w \in T^{[q]}(w)\right\}$.

Remark 4.5. If $(X, T)$ is a single-valued dynamic system, then Theorems 4.4(B) and 4.4(C) are identical.

\section{Examples}

In this section, we present some examples illustrating the concepts introduced so far.

In Example 5.1, we define two $2 p$-families in quasigauge spaces. 
Example 5.1. Let the family $D=\left\{p_{\alpha}: \alpha \in \mathcal{A}\right\}$ of quasipseudometrics $p_{\alpha}: X \times X \rightarrow[0, \infty)$, $\alpha \in \mathcal{A}$ be a quasigauge on $X$ and let $(X, D)$ be a quasigauge space.

(I) The family $D$ is a $2 p$-family on $X$ (see Remark 3.2(b)).

(II) Let $X$ contain at least two different points. Let the set $E \subset X$ containing at least two different points be arbitrary and fixed, and let $\left\{c_{\alpha}\right\}_{\alpha \in \mathcal{A}}$ satisfy $\forall_{\alpha \in \mathcal{A}}\left\{\delta_{\alpha}(E)<c_{\alpha}\right\}$, where $\forall_{\alpha \in \mathcal{A}}\left\{\delta_{\alpha}(E)=\sup \left\{p_{\alpha}(x, y): x, y \in E\right\}\right\}$. Let the family $\partial=\left\{J_{\alpha}: \alpha \in \mathscr{A}\right\}$, $J_{\alpha}: X \times X \rightarrow[0, \infty), \alpha \in \mathcal{A}$ be defined by the following formula:

$$
J_{\alpha}(x, y)=\left\{\begin{array}{ll}
p_{\alpha}(x, y) & \text { if } E \cap\{x, y\}=\{x, y\} \\
c_{\alpha} & \text { if } E \cap\{x, y\} \neq\{x, y\}
\end{array} \quad x, y \in X\right.
$$

We show that the family $\partial$ is a $2 p$-family on $X$.

Indeed, we see that the condition (21) does not hold only if there exist some $\alpha \in \mathcal{A}$ and $x, y, z \in X$ such that $J_{\alpha}(x, y)=c_{\alpha}, J_{\alpha}(x, z)=p_{\alpha}(x, z), J_{\alpha}(z, y)=p_{\alpha}(z, y)$, and $p_{\alpha}(x, z)+$ $p_{\alpha}(z, y)<c_{\alpha}$. However, then we conclude that there exists $v \in\{x, y\}$ such that $v \notin E$ and $x, y, z \in E$, which is impossible. Therefore, $\forall_{\alpha \in \mathscr{A}} \forall_{x, y, z \in X}\left\{J_{\alpha}(x, y) \leqslant J_{\alpha}(x, z)+J_{\alpha}(z, y)\right\}$, that is, the condition (21) holds.

For proving that (22) holds, we assume that the sequences $\left\{w_{m}\right\}$ and $\left\{v_{m}\right\}$ in $X$ satisfy (3.1) and (3.2). Then, in particular, (3.2) yields

$$
\forall_{\alpha \in \mathcal{A}} \forall_{0<\varepsilon<c_{\alpha}} \exists_{m_{0}=m_{0}(\alpha) \in \mathbb{N}} \forall_{m \geqslant m_{0}}\left\{J_{\alpha}\left(w_{m}, v_{m}\right)<\varepsilon\right\} .
$$

By (5.2) and (5.1), denoting $m^{\prime}=\min \left\{m_{0}(\alpha): \alpha \in \mathcal{A}\right\}$, we conclude that

$$
\forall_{m \geqslant m^{\prime}}\left\{E \cap\left\{w_{m}, v_{m}\right\}=\left\{w_{m}, v_{m}\right\}\right\}
$$

From (5.3), definition of 2 , and (5.2), we get

$$
\forall_{\alpha \in \mathcal{A}} \forall_{0<\varepsilon<c_{\alpha}} \exists_{m^{\prime} \in \mathbb{N}} \forall_{m \geqslant m^{\prime}}\left\{p_{\alpha}\left(w_{m}, v_{m}\right)=J_{\alpha}\left(w_{m}, v_{m}\right)<\varepsilon\right\} .
$$

The result is that the sequences $\left\{w_{m}\right\}$ and $\left\{v_{m}\right\}$ satisfy (3.3). Therefore, the property (22) holds.

The following example illustrates Theorem 4.4(A) in not Hausdorff quasigauge space.

Example 5.2. Let $X=[0,1 / 2] \cup\{3 / 4,1\} \subset \mathbb{R}$, and let $T: X \rightarrow 2^{X}$ be of the form

$$
T(x)= \begin{cases}\{1\} & \text { for } x=0 \\ {[0,(1 / 2) x]} & \text { for } x \in(0,1 / 2] \\ \{0,1\} & \text { for } x=3 / 4 \\ \{0,3 / 4\} & \text { for } x=1 .\end{cases}
$$


Let the map $p: X \times X \rightarrow[0, \infty)$ be defined by the formula

$$
p(x, y)=\left\{\begin{array}{ll}
0 & \text { if } x \geqslant y, \\
1 & \text { if } x<y,
\end{array} \quad(x, y) \in X \times X,\right.
$$

and let $D=\{p\}$; this map $p$ is a modification of a map $p$ due to Reilly et al. [3, Example 1]. For a set $E=[0,1 / 2]$, let the family $\partial=\{J: X \times X \rightarrow[0, \infty)\}$ be defined as follows

$$
J(x, y)=\left\{\begin{array}{ll}
p(x, y) & \text { if } E \cap\{x, y\}=\{x, y\}, \\
2 & \text { if } E \cap\{x, y\} \neq\{x, y\}
\end{array} \quad x, y \in X\right.
$$

and let $\Gamma=\{\gamma\}$, where $\gamma: X \rightarrow[0, \infty)$ is of the form $\gamma(x)=x, x \in X$.

(a) The map $p: X \times X \rightarrow[0, \infty)$ is a quasipseudometric on $X$.

Indeed, we have that $p(x, x)=0$ since $x \geqslant x$, and thus $(D 1)$ holds. Also $(D 2)$ is satisfied since we obtain the following. (1) If $x \geqslant z$, then $p(x, z)=0 \leqslant p(x, y)+p(y, z)$ for each $y \in X$. (2) If $x<z$, then $p(x, z)=1$, and suppose that $p(x, y)=p(y, z)=0$ for some $y \in X$, then $x \geqslant y$ and $y \geqslant z$ which implies $x \geqslant z$. This is absurd because $x<z$. Consequently, $\forall y \in X\{p(x, y)=1 \vee p(y, z)=1\}$.

(b) Quasigauge space $(X, P)$ is a left sequentially complete.

Indeed, let $\left(w_{m}: m \in \mathbb{N}\right)$ be left $P$-Cauchy sequence in $X$ (i.e., let (2.2) hold), and let $\eta_{0}, 0<\eta_{0}<1$, be arbitrary and fixed. Then, by $(2.2)$, we get

$$
\exists_{w_{0} \in X} \exists_{k_{0} \in \mathbb{N}} \forall_{m} \geqslant k_{0}\left\{p\left(w_{0}, w_{m}\right)<\eta_{0}<1\right\} .
$$

Now, if $\varepsilon_{0}>0$ is arbitrary and fixed, then, by (5.6) and (5.8), for each $m \geqslant k_{0}$, $p\left(w_{0}, w_{m}\right)=0<\varepsilon_{0}$.

Hence, we conclude that $\exists_{w_{0} \in X} \forall_{\varepsilon>0} \exists_{k \in \mathbb{N}} \forall_{m \geqslant k}\left\{p\left(w_{0}, w_{m}\right)<\varepsilon\right\}$. This gives that $\left(w_{m}: m \in\right.$ $\mathbb{N})$ left converges to $w_{0}$.

(c) Quasigauge space $(X, D)$ is a left $K$ sequentially complete.

This follows from (b) and Remark 2.7(c).

(d) The family $2=\{J: X \times X \rightarrow[0, \infty)\}$ is a $\partial_{p}$-family on $X$.

This is the consequence of Example 5.1(II). Let us observe, additionally, that $\mathrm{cl}_{\mathrm{L}}(E)=X$.

(e) $T$ is weak $\left(2, \Gamma ; X_{0}\right)$ dissipative on $X$, where $X_{0}=(0,1 / 2]$.

Indeed, let $x \in(0,1 / 2]$ be arbitrary and fixed. We have that $T^{[2]}(x)=[0,(1 / 4) x] \cup\{1\}$ and $T^{[m]}(x)=\left[0,\left(1 / 2^{m}\right) x\right] \cup\{3 / 4,1\}$ for $m \geqslant 3$. Thus, there exists a dynamic process $\left(w_{m}\right.$ : $m \in\{0\} \cup \mathbb{N})$ given by the formula $w_{0}=x, w_{m}=\left(1 / 2^{m}\right) x, m \in \mathbb{N}$, such that $J\left(w_{0}, w_{1}\right)=$ $p(x,(1 / 2) x)=0 \leqslant x-x / 2=\gamma\left(w_{0}\right)-\gamma\left(w_{1}\right)$ and $\forall_{m \in \mathbb{N}}\left\{J\left(w_{m}, w_{m+1}\right)=p\left(w_{m}, w_{m+1}\right)=0 \leqslant\right.$ $\left.\gamma\left(w_{m}\right)-\gamma\left(w_{m+1}\right)\right\}$. This gives that the dynamic process $\left(w_{m}: m \in\{0\} \cup \mathbb{N}\right)$ satisfies (1.1) and (3.4). Consequently, $x \in X_{0}$. Therefore, we proved that $(0,1 / 2] \subset X_{0}$.

Now, we show that $X_{0} \subset(0,1 / 2]$. Suppose that $X_{0} \cap\{X \backslash(0,1 / 2]\} \neq \emptyset$ then there exists $x \in X_{0}$ such that $x \notin(0,1 / 2]$, and we consider the following three cases. 
Case 1. If $x=0$, then each dynamic process $\left(w_{m}: m \in\{0\} \cup \mathbb{N}\right)$ starting at $w_{0}=0$ of the system $(X, T)$ satisfies $w_{1}=1=T\left(w_{0}\right)$ and $0<J\left(w_{0}, w_{1}\right)=2>-1=0-1=\gamma\left(w_{0}\right)-\gamma\left(w_{1}\right)$, that is, (3.4) does not hold.

Case 2. Let $x=3 / 4$ and let $\left(w_{m}: m \in\{0\} \cup \mathbb{N}\right)$ be an arbitrary and fixed dynamic process starting at $w_{0}=3 / 4$ of the system $(X, T)$. If $w_{1}=1$, then $0<J\left(w_{0}, w_{1}\right)=2>3 / 4-1=$ $\gamma\left(w_{0}\right)-\gamma\left(w_{1}\right)$, that is, (3.4) does not hold. If $w_{1}=0$, then $w_{2}=1$ and $0<J\left(w_{1}, w_{2}\right)=2>$ $0-1=\gamma\left(w_{1}\right)-\gamma\left(w_{2}\right)$, that is, (3.4) does not hold.

Case 3. Let $x=1$ and let $\left(w_{m}: m \in\{0\} \cup \mathbb{N}\right)$ be an arbitrary and fixed dynamic process starting at $w_{0}=1$ of the system $(X, T)$. If $w_{1}=0$, then $w_{2}=1$ and $0<J\left(w_{1}, w_{2}\right)=2>0-1=$ $\gamma\left(w_{1}\right)-\gamma\left(w_{2}\right)$, that is, (3.4) does not hold. If $w_{1}=3 / 4$, then $0<J\left(w_{0}, w_{1}\right)=2>1-3 / 4=$ $1 / 4=\gamma\left(w_{0}\right)-\gamma\left(w_{1}\right)$, that is, (3.4) does not hold.

Consequently, $X_{0}=(0,1 / 2]$.

(f) We have that $\mathrm{cl}_{\mathrm{L}}\left(\mathrm{X}_{0}\right)=(0,1 / 2] \cup\{3 / 4,1\}$.

First, we show that $X \backslash\{0\} \subset \mathrm{cl}_{\mathrm{L}}\left(X_{0}\right)$, that is, by Definition 4.2(ii), for each $w \in X \backslash\{0\}$, there exists a sequence $\left(w_{m}: m \in \mathbb{N}\right) \subset X_{0}$ which left converges to $w$. Indeed, if $w \in X \backslash\{0\}$ is arbitrary and fixed, then for the sequence defined by $w_{1}=w$ and $w_{m}=c^{m} w$ for $m \geqslant 2$, where $0<c<1 / 2$ is arbitrary and fixed, we get that $\exists_{k \in \mathbb{N}} \forall_{m \geqslant k}\left\{w_{m}<w\right\}$ and $\left(w_{m}: m \in \mathbb{N}\right) \subset X_{0}$. Consequently, for arbitrary and fixed $\varepsilon>0$, by (5.6), we have $\forall_{m \geqslant k}\left\{p\left(w, w_{m}\right)=0<\varepsilon\right\}$. Therefore, $\left(w_{m}: m \in \mathbb{N}\right)$ left converges to $w$. By Definition 4.2(ii), $w \in \mathrm{cl}_{\mathrm{L}}\left(\mathrm{X}_{0}\right)$. This gives that $X \backslash\{0\} \subset \mathrm{cl}_{\mathrm{L}}\left(\mathrm{X}_{0}\right)$.

Now, we show that $\mathrm{cl}_{\mathrm{L}}\left(X_{0}\right) \subset X \backslash\{0\}$, that is, $0 \notin \mathrm{cl}_{\mathrm{L}}\left(X_{0}\right)$. Otherwise, $0 \in \mathrm{cl}_{\mathrm{L}}\left(X_{0}\right)$, and thus there exists a sequence $\left(w_{m}: m \in \mathbb{N}\right) \subset X_{0}$ which left converges to 0 , that is, $\forall_{\varepsilon>0} \exists_{k \in \mathbb{N}} \forall_{m \in \mathbb{N} ; k \leqslant m}\left\{p\left(0, w_{m}\right)<\varepsilon\right\}$. This is absurd because $\forall_{m \in \mathbb{N}}\left\{0<w_{m}\right\}$ which, by (5.6), gives $\forall_{m \in \mathbb{N}}\left\{p\left(0, w_{m}\right)=1\right\}$.

(g) Theorem 4.4 $)$ holds.

Indeed, by the considerations in (e), if $x \in(0,1 / 2]=X_{0}$, then $\mathcal{K}_{2}(T, x) \neq \emptyset$, and if $\left(w_{m}: m \in\{0\} \cup \mathbb{N}\right) \in \mathcal{K}_{2}(T, x)$ is arbitrary and fixed, then, by Cases $1-3$ in $(\mathrm{e}), \forall_{m \in \mathbb{N}}\left\{w_{m} \in\right.$ $\left.\left[0,\left(1 / 2^{m}\right) x\right]\right\}$. Therefore, from $(\mathrm{f}),\left(w_{m}: m \in\{0\} \cup \mathbb{N}\right)$ left converges to each point $w \in W=$ $\mathrm{cl}_{\mathrm{L}}\left(\mathrm{X}_{0}\right)$.

(h) The map $T$ is not left quasiclosed in $X$.

Indeed, if $\left(w_{m}: m \in \mathbb{N}\right) \subset X$ is such that $\forall_{m \in \mathbb{N}}\left\{w_{m}=0\right\}$, then $W=[0,1 / 2] \cup\{3 / 4,1\}$. Next, we see that if $\left(v_{m}: m \in \mathbb{N}\right)$ satisfies $\forall_{m \in \mathbb{N}}\left\{v_{m} \in T\left(w_{m}\right)\right\}$, then $\forall_{m \in \mathbb{N}}\left\{v_{m}=1\right\}$ which gives $V=\{1\}$. Consequently, $\exists_{v \in V} \forall_{w \in W}\{v \in T(w)\}$ does not hold because $w=1 \in W$ and $V \cap T(1)=\{1\} \cap\{0,3 / 4\}=\emptyset$.

(i) The map $T^{[2]}$ is not left quasiclosed in $X$.

Indeed, we have that

$$
T^{[2]}(x)= \begin{cases}\{0,3 / 4\} & \text { for } x=0 \\ {[0,(1 / 4) x] \cup\{1\}} & \text { for } x \in(0,1 / 2] \\ \{0,3 / 4,1\} & \text { for } x=3 / 4 \\ \{0,1\} & \text { for } x=1 .\end{cases}
$$


Thus, if $\left(w_{m}: m \in \mathbb{N}\right)$ is such that $\forall_{m \in \mathbb{N}}\left\{w_{m}=0\right\}$, then $W=[0,1 / 2] \cup\{3 / 4,1\}$, and if $\left(v_{m}\right.$ : $m \in \mathbb{N}$ ) satisfying $\forall_{m \in \mathbb{N}}\left\{v_{m} \in T^{[2]}\left(w_{m}\right)\right\}$ is of the form $\forall_{m \in \mathbb{N}}\left\{v_{m}=3 / 4\right\}$, then $V=\{3 / 4,1\}$. Consequently, $\exists_{v \in V} \forall_{w \in W}\left\{v \in T^{[2]}(w)\right\}$ does not hold because (I) if $v=3 / 4 \in V$, then, for $w=1 \in W$, we have $\{3 / 4\} \cap T^{[2]}(1)=\{3 / 4\} \cap\{0,1\}=\emptyset$, (II) if $v=1 \in V$, then, for $w=0 \in W$, we have $\{1\} \cap T^{[2]}(0)=\{1\} \cap\{0,3 / 4\}=\emptyset$.

(j) The map $T^{[3]}$ is left quasiclosed in $X$.

Indeed, we have that

$$
T^{[3]}(x)= \begin{cases}\{0,1\} & \text { for } x=0 \\ {[0,(1 / 8) x] \cup\{3 / 4,1\}} & \text { for } x \in(0,1 / 2] \\ \{0,3 / 4,1\} & \text { for } x=3 / 4 \\ \{0,3 / 4,1\} & \text { for } x=1,\end{cases}
$$

and if $\left(w_{m}: m \in \mathbb{N}\right)$ is an arbitrary and fixed sequence in $X, W$ is a set of all left limit points of $\left(w_{m}: m \in \mathbb{N}\right),\left(v_{m}: m \in \mathbb{N}\right)$ is an arbitrary and fixed sequence satisfying $\forall_{m \in \mathbb{N}}\left\{v_{m} \in\right.$ $\left.T^{[3]}\left(w_{m}\right)\right\}$, and $V$ is a set of all left limit points of $\left(v_{m}: m \in \mathbb{N}\right)$, then we see that $\forall_{x \in X}\{1 \in$ $\left.T^{[3]}(x)\right\}$ and $1 \in V$. Consequently, $\exists_{v=1 \in V} \forall_{w \in W}\left\{v \in T^{[3]}(w)\right\}$.

(k) The assumptions of Theorem $4.4\left(A_{2}\right)$ hold for $q=3$.

This is the consequence of $(\mathrm{h})-(\mathrm{j})$. The assertion is that $\left\{w \in W \subset \mathrm{cl}_{\mathrm{L}}\left(X_{0}\right): w \in\right.$ $\left.T^{[q]}(w)\right\}=\{3 / 4,1\} \subset \operatorname{Fix}\left(T^{[3]}\right)$, that is, $\{3 / 4,1\} \subset \operatorname{Per}(T)$ for $q=3$.

(1) The map $T$ is not $(2, \Gamma)$ dissipative on $X$ and not strictly $(2, \Gamma)$ dissipative on $X$.

This is the consequence of Cases 1-3 in (e).

(m) For any $\Gamma, T$ is not $(2, \Gamma)$ dissipative on $X$.

Indeed, suppose that there exists $\Gamma=\{\gamma\}$ such that $\gamma: X \rightarrow[0, \infty)$ and that $T$ is $(2, \Gamma)$ dissipative on $X$. Then, for a dynamic process $\left(w_{m}: m \in\{0\} \cup \mathbb{N}\right)$ starting at $w_{0}=3 / 4$ defined by $w_{1}=0 \in T\left(w_{0}\right), w_{2}=1 \in T\left(w_{1}\right), w_{3 m+1}=0 \in T\left(w_{3 m}\right), w_{3 m+2}=1 \in T\left(w_{3 m+1}\right)$, and $w_{3 m}=3 / 4 \in T\left(w_{3 m-1}\right)$ for $m \in \mathbb{N}$, we have $0<J\left(w_{0}, w_{1}\right)=2 \leqslant \gamma\left(w_{0}\right)-\gamma\left(w_{1}\right), 0<$ $J\left(w_{1}, w_{2}\right)=2 \leqslant \gamma\left(w_{1}\right)-\gamma\left(w_{2}\right)$, and $0<J\left(w_{2}, w_{3}\right)=2 \leqslant \gamma\left(w_{2}\right)-\gamma\left(w_{3}\right)=\gamma\left(w_{2}\right)-\gamma\left(w_{0}\right)$. Hence, $\gamma\left(w_{0}\right)<\gamma\left(w_{2}\right)<\gamma\left(w_{1}\right)<\gamma\left(w_{0}\right)$, which is impossible.

(n) For any $\Gamma, T$ is not strictly $(2, \Gamma)$ dissipative on $X$.

This is the consequence of $(\mathrm{m})$ and Remark 3.6(b).

(o) Quasigauge space $(X, P)$ is not Hausdorff.

Indeed, for $x_{0}, y_{0} \in X$ such that $x_{0}>y_{0}$, there exists $z_{0} \in X$ such that $z_{0} \leqslant y_{0}$. Then, for each $\varepsilon, \eta>0$, by (5.6), we have that $p\left(x_{0}, z_{0}\right)=0<\varepsilon$ and $p\left(y_{0}, z_{0}\right)=0<\eta$, which implies that $z_{0} \in B\left(x_{0}, \varepsilon\right) \cap B\left(y_{0}, \eta\right)$.

The following example illustrates Theorems 4.4 (B) and 4.4 (C) in quasimetric space.

Example 5.3. Let $X=\mathbb{N} \subset \mathbb{R}$, let $T: X \rightarrow X$ be defined by

$$
T(x)= \begin{cases}1 & \text { if } x=1 \\ x-1 & \text { if } x=2 k, k \in \mathbb{N} \\ x-2 & \text { if } x=2 k+1, k \in \mathbb{N}\end{cases}
$$


and let $p: X \times X \rightarrow[0, \infty)$ be defined by the formulae

$$
\begin{aligned}
& p(m, n)=0 \quad \text { if } m=n, \\
& p(m, n)=m^{-1} \quad \text { if } m<n \text { and } m \text { is even and } n \text { is odd, } \\
& p(m, n)=1 \quad \text { otherwise; }
\end{aligned}
$$

this map $p$ is a modification of a map $p$ due to Reilly et al. [3, Example 5].

Let $D=\{p\}$, let $\partial=\{J\}$ where $J(m, n)=p(m, n)$ for $m, n \in \mathbb{N}$, and let $\Gamma=\{\gamma\}$ where $\gamma: X \rightarrow[0, \infty)$ is of the form

$$
\gamma(x)=x, \quad x \in X
$$

(a) The map $p$ is quasimetric on $\mathrm{X}$.

This is the consequence of the following useful observations.

Case 1. If $m=n$, then $p(m, n)=0 \leqslant p(m, k)+p(k, n)$ for each $k \in X$.

Case 2. If $m>n$ and $k \in X$, then $p(m, n) \leqslant p(m, k)+p(k, n)$. Indeed, by (5.14), $p(m, n)=1$. On the other hand, $k \leqslant n$ means that $k<m$. By (5.14), $k<m$ implies $p(m, k)=1$ and $n<k$ implies $p(k, n)=1$. Hence, for each $k \in X, 1 \leqslant p(m, k)+p(k, n)$.

Case 3. If $m<n$ and $k<m$ or $n<k$, then $p(m, n) \leqslant p(m, k)+p(k, n)$. Indeed, by (5.12)-(5.14), $p(m, n) \leqslant 1$. Next, for $k<m$ or $n<k$, we have $1 \leqslant p(m, k)+p(k, n)$ since, by $(5.14), k<m$ implies $p(m, k)=1$ and $n<k$ implies $p(k, n)=1$.

Case 4. If $m<n$ and $m \leqslant k \leqslant n$, then $p(m, n) \leqslant p(m, k)+p(k, n)$ since the following five properties $\left(\mathrm{IV}_{1}\right)-\left(\mathrm{IV}_{2}\right)$ are satisfied.

$\left(\mathrm{IV}_{1}\right)$ Let $k=m$ or $k=n$ then $p(m, n)=p(m, k)+p(k, n)$.

$\left(\mathrm{IV}_{2}\right)$ Let $m$ be even, let $n$ be odd and let $m<k<n$ then, by (5.12), $p(m, n)=m^{-1}$. If $k$ is odd, then, by (5.13), $p(m, k)=m^{-1}$ and, by (5.14), $p(k, n)=1$. If $k$ is even, then, by (5.13), $p(k, n)=k^{-1}$ and, by (5.14), $p(m, k)=1$. Consequently, $p(m, n)<$ $p(m, k)+p(k, n)$.

$\left(\mathrm{IV}_{3}\right)$ Let $m$ and $n$ be even, and let $m<k<n$. Then, by (5.14), $p(m, n)=1$. If $k$ is odd, then, by (5.13), $p(m, k)=m^{-1}$ and, by (5.14), $p(k, n)=1$. If $k$ is even, then, by (5.14), $p(k, n)=p(m, k)=1$. Consequently, $p(m, n)<p(m, k)+p(k, n)$.

$\left(\mathrm{IV}_{4}\right)$ Let $m$ and $n$ be odd, and let $m<k<n$. Then, by (5.14), $p(m, n)=1$. If $k$ is odd, then, by (5.14), $p(m, k)=p(k, n)=1$. If $k$ is even, then, by (5.13), $p(k, n)=k^{-1}$ and, by $(5.14), p(m, k)=1$. Consequently, $p(m, n)<p(m, k)+p(k, n)$.

$\left(\mathrm{IV}_{5}\right)$ Let $m$ be odd, let $n$ be even, and let $m<k<n$. Then, by (5.14), $p(m, n)=p(m, k)=$ $p(k, n)=1$. Consequently, $p(m, n)<p(m, k)+p(k, n)$. 
(b) The family 2 is a $2 p$-family.

This follows from Example 5.1(I).

(c) $(X, P)$ is a left $K$ sequentially complete quasimetric space.

Indeed, we see that only sequences $\left(w_{m}: m \in \mathbb{N}\right)$ in $X$ satisfying

$$
\exists_{k \in \mathbb{N}} \exists_{w \in \mathbb{N}} \forall_{m \geqslant k}\left\{w_{m}=w\right\}
$$

are left- $(\mathcal{D}, K-)$ Cauchy sequences in $X$, that is, satisfy (2.1). Further, each sequence $\left(w_{m}\right.$ : $m \in \mathbb{N}$ ) in $X$ satisfying (5.16) is left convergent in $X$, that is, satisfies (2.3). Consequently, $(X, D)$ is a left $K$ sequentially complete.

(d) $(X, D)$ is not a left sequentially complete quasimetric space.

Indeed, using (5.13), we see that the sequence $\left(w_{m}: m \in \mathbb{N}\right)$ in $X$ of the form $w_{m}=$ $2 m-1, m \in \mathbb{N}$, satisfies $\forall_{\varepsilon>0} \exists_{w=2 m_{0} \in X} \forall_{m \in \mathbb{N}}\left\{p\left(w, w_{m}\right)=1 /\left(2 m_{0}\right)<\varepsilon\right\}$, and thus $(2 m-1: m \in \mathbb{N})$ is left $D$-Cauchy sequence in $X$ (i.e., satisfies (2.2)).

Now, suppose that for this sequence the condition (2.3) holds, that is, that $\exists_{w \in \mathbb{N}} \forall_{\varepsilon>0} \exists_{k \in \mathbb{N}} \forall_{m \in \mathbb{N} ; k \leqslant m}\left\{p\left(w, w_{m}\right)<\varepsilon\right\}$. It is clear that then $\exists_{s \in \mathbb{N}} \forall_{m \geqslant s}\left\{w<w_{m}\right\}$. Hence, since, for each $m \in \mathbb{N}, w_{m}$ is odd, using (5.13) and (5.14), we obtain that

$$
\exists_{w \in \mathbb{N}} \forall_{\varepsilon>0} \forall_{m \geqslant s}\left\{\varepsilon>p\left(w, w_{m}\right)= \begin{cases}1 / w & \text { if } w \text { is even } \\ 1 & \text { if } w \text { is odd }\end{cases}\right.
$$

which is impossible.

(e) The map $T$ is strictly $(2, \Gamma)$ dissipative on $X$.

Indeed, let $x \in X$ be arbitrary and fixed, and consider the following three cases.

Case 1. If $x=1$, then there exists a unique generalized sequence of iterations $\left(w_{m}: m \in\{0\} \cup\right.$ $\mathbb{N})$ starting at $w_{0}=x$ of the system $(X, T)$, given by the formula $w_{m}=1, m \in\{0\} \cup \mathbb{N}$. Hence, by (5.12) and (5.15), we have $\forall_{m \in\{0\} \cup \mathbb{N}}\left\{J\left(w_{m}, w_{m+1}\right)=p\left(w_{m}, w_{m+1}\right)=0 \leqslant \gamma\left(w_{m}\right)-\gamma\left(w_{m+1}\right)=0\right\}$. Therefore, the sequence $\left(w_{m}: m \in\{0\} \cup \mathbb{N}\right)$ satisfies (1.2) and (3.4) and left converges to $w=1$.

Case 2. If $x=2 k$, where $k \in \mathbb{N}$, then there exists a unique generalized sequence of iterations $\left(w_{m}: m \in\{0\} \cup \mathbb{N}\right)$ starting at $w_{0}=x$ of the system $(X, T)$ defined by the formula $w_{0}=2 k$, $w_{m}=2 k-(2 m-1)=T^{[m]}\left(w_{0}\right)$ for $m \in\{1,2,3, \ldots, k-1\}$, and $w_{m}=1=T^{[m]}\left(w_{0}\right)$ for $m \geqslant k$. Hence, by (5.14) and (5.15), we get that $J\left(w_{0}, w_{1}\right)=p(2 k, 2 k-1)=1 \leqslant 2 k-(2 k-1)=$ $\gamma\left(w_{0}\right)-\gamma\left(w_{1}\right)$ and $\forall_{m \in\{1, \ldots, k-1\}}\left\{J\left(w_{m}, w_{m+1}\right)=p\left(w_{m}, w_{m+1}\right)=1 \leqslant 2=w_{m}-w_{m+1}=\gamma\left(w_{m}\right)-\right.$ $\left.\gamma\left(w_{m+1}\right)\right\}$ and, by (5.12) and (5.15), we get that $\forall_{m \geqslant k}\left\{J\left(w_{m}, w_{m+1}\right)=p\left(w_{m}, w_{m+1}\right)=0 \leqslant\right.$ $\left.\gamma\left(w_{m}\right)-\gamma\left(w_{m+1}\right)\right\}$. Therefore, the sequence $\left(w_{m}: m \in\{0\} \cup \mathbb{N}\right)$ satisfies (1.2) and (3.4) and left converges to $w=1$.

Case 3. If $x=2 k+1$, where $k \in \mathbb{N}$, then there exists a unique generalized sequence of iterations $\left(w_{m}: m \in\{0\} \cup \mathbb{N}\right)$ starting at $w_{0}=x$ of the system $(X, T)$ defined by the formula $w_{m}=2 k-$ $(2 m-1)=T^{[m]}\left(w_{0}\right)$ for $m \in\{0,1,2,3, \ldots, k-1\}$ and $w_{m}=1=T^{[m]}\left(w_{0}\right)$ for $m \geqslant k$. Hence, by 
(5.14) and (5.15), we get that $J\left(w_{0}, w_{1}\right)=p(2 k+1,2 k-1)=1 \leqslant 2 k+1-(2 k-1)=\gamma\left(w_{0}\right)-\gamma\left(w_{1}\right)$ and $\forall_{m \in\{1, \ldots, k-1\}}\left\{J\left(w_{m}, w_{m+1}\right)=p\left(w_{m}, w_{m+1}\right)=1 \leqslant 2=w_{m}-w_{m+1}=\gamma\left(w_{m}\right)-\gamma\left(w_{m+1}\right)\right\}$ and, by (5.12) and (5.15), we get that $\forall_{m \geqslant k}\left\{J\left(w_{m}, w_{m+1}\right)=p\left(w_{m}, w_{m+1}\right)=0 \leqslant \gamma\left(w_{m}\right)-\gamma\left(w_{m+1}\right)\right\}$. Therefore, the sequence $\left(w_{m}: m \in\{0\} \cup \mathbb{N}\right)$ satisfies (1.2) and (3.4) and left converges to $w=1$.

The above implies that $T$ is strictly $(2, \Gamma)$ dissipative on $X$.

(f) The assertion of Theorem $4.4\left(C_{1}\right)$ holds.

From considerations in (e), it follows that, for each $x \in X$, a generalized sequence of iterations $\left(w_{m}: m \in\{0\} \cup \mathbb{N}\right)$ starting at $w_{0}=x$ of the system $(X, T)$ satisfies $\left\{\left(w_{m}: m \in\right.\right.$ $\{0\} \cup \mathbb{N})\}=S_{2}(T, x)$ and left converges to $w \in W=\{1\} \subset X$.

(g) The map $T$ is left quasiclosed in $X$.

Indeed, in $X$ only sequences $\left(w_{m}: m \in \mathbb{N}\right)$ satisfying the condition $\exists_{k \in \mathbb{N}} \exists_{w \in X} \forall_{m ; k \leqslant m}\left\{w_{m}=w\right\}$ are left convergent to $w$ and $W=\{w\}$. Further, if $\forall_{m \in \mathbb{N}}\left\{v_{m}=\right.$ $\left.T\left(w_{m}\right)\right\}$, then a sequence $\left(v_{m}: m \in \mathbb{N}\right)$ left converges to $v=T(w)$ and $V=\{v\}$ where

$$
V= \begin{cases}\{1\} & \text { if } w=1 \\ \{w-1\} & \text { if } w=2 k, k \in \mathbb{N} \\ \{w-2\} & \text { if } w=2 k+1, k \in \mathbb{N}\end{cases}
$$

By Definition 4.2(i), the map $T$ is left quasiclosed in $X$.

(h) The assertion of Theorem $4.4\left(C_{2}\right)$ holds.

Indeed, by (e)-(g), for each $x \in X$, there exists a generalized sequence of iterations $\left(w_{m}: m \in\{0\} \cup \mathbb{N}\right)$ starting at $w_{0}=x$ of the system $(X, T)$ satisfying $\exists_{k \in \mathbb{N}} \forall_{m ; k \leqslant m}\left\{w_{m}=1\right\}$ and $\left\{\left(w_{m}: m \in\{0\} \cup \mathbb{N}\right)\right\}=\mathcal{S}_{2}(T, x)$, left converging to $w \in W=\{1\}$, for which $\left\{\left(v_{m}: m \in\right.\right.$ $\{0\} \cup \mathbb{N})\}$, where $\forall_{m \in \mathbb{N}}\left\{v_{m}=T\left(w_{m}\right)\right\}$ satisfies $\forall_{m ; k \leqslant m}\left\{v_{m}=1\right\}$, left converges to $v \in V=\{1\}$, and $w=1$ is the fixed point of $T$. holds.

From (a)-(h), it follows that Theorem 4.4(C) (also Theorem 4.4(B) by Remark 4.5)

The following example shows that in Theorem 4.4 the assumptions in $\left(\mathrm{A}_{2}\right),\left(\mathrm{B}_{2}\right)$, and $\left(C_{2}\right)$ (i.e., the assumptions that the map $T^{[q]}$ is left quasiclosed in $X$ for some $q \in \mathbb{N}$ ) are essential.

Example 5.4. Let $X=[0,1]$, and let $D=\{p\}$ where $p(x, y)=|x-y|, x, y \in X$. Then, the family $\partial=\{J\}$, where $J=p$, is a $2 p$-family. Let $T: X \rightarrow 2^{X}$ be of the form

$$
T(x)= \begin{cases}\{1\} & \text { if } x=0 \\ {[0,(1 / 2) x]} & \text { if } x \in(0,1) \\ \{1\} & \text { if } x=1\end{cases}
$$


and let $\Gamma=\{\gamma: X \rightarrow[0, \infty)\}$, where $\gamma$ is of the form

$$
\gamma(x)= \begin{cases}1 & \text { if } x=0 \\ x & \text { if } x \in(0,1), x \in X \\ 0 & \text { if } x=1\end{cases}
$$

(a) Theorem $4.4\left(B_{1}\right)$ holds.

We show that the map $T$ is $(2, \Gamma)$ dissipative. Indeed, let $x \in X$ be arbitrary and fixed. We consider three cases.

Case 1. If $x=0$, then there exists only one dynamic process $\left(w_{m}: m \in\{0\} \cup \mathbb{N}\right)$ starting at $w_{0}=0$. This dynamic process is of the form $\forall_{m \in \mathbb{N}}\left\{w_{m}=1=T\left(w_{m-1}\right)=T^{[m]}\left(w_{0}\right)\right\}$ and satisfies $J\left(w_{0}, w_{1}\right)=1 \leqslant 1-0=\gamma(0)-\gamma(1)$ and $\forall_{m \in \mathbb{N}}\left\{J\left(w_{m}, w_{m+1}\right)=0 \leqslant \gamma\left(w_{m}\right)-\gamma\left(w_{m+1}\right)\right\}$.

Case 2. If $x \in(0,1)$, then each dynamic process $\left(w_{m}: m \in\{0\} \cup \mathbb{N}\right)$ starting at $w_{0}=x$ satisfies

$$
\forall_{m \in \mathbb{N}}\left\{w_{m} \in(0,1)\right\}
$$

or

$$
\exists_{k \in \mathbb{N}}\left\{w_{k}=0\right\} .
$$

If (5.21) holds, then $\forall_{m \in \mathbb{N}}\left\{w_{m} \in\left(0,(1 / 2) w_{m-1}\right] \subset T\left(w_{m-1}\right) \subset\left[0,\left(1 / 2^{m}\right) w_{0}\right] \cup\{1\}=\right.$ $\left.T^{[m]}\left(w_{0}\right)\right\}$ and $\forall_{m \in\{0\} \cup \mathbb{N}}\left\{0<J\left(w_{m}, w_{m+1}\right)=w_{m}-w_{m+1}=\gamma\left(w_{m}\right)-\gamma\left(w_{m+1}\right)\right\}$.

If (5.22) holds, then $\forall_{m \leqslant k}\left\{w_{m} \in T\left(w_{m-1}\right)=\left[0,(1 / 2) w_{m-1}\right] \subset\left[0,\left(1 / 2^{m}\right) w_{0}\right] \cup\{1\}=\right.$ $\left.T^{[m]}\left(w_{0}\right)\right\}, w_{k+1} \in\{1\}=T(0)=T\left(w_{k}\right) \subset T^{[k]}\left(w_{0}\right)$, and $\forall_{m>k+1}\left\{w_{m} \in\{1\}=T\left(w_{m-1}\right) \subset\right.$ $\left.T^{[m]}\left(w_{0}\right)\right\}$. The above implies that $\forall_{m<k}\left\{w_{m} \in\left(0,(1 / 2) w_{m-1}\right]\right.$ and $J\left(w_{m}, w_{m+1}\right)=w_{m}-w_{m+1}=$ $\left.\gamma\left(w_{m}\right)-\gamma\left(w_{m+1}\right)\right\}, J\left(w_{k}, w_{k+1}\right)=\left|w_{k}-w_{k+1}\right|=|0-1|=1=1-0=\gamma\left(w_{k}\right)-\gamma\left(w_{k+1}\right)$, and $\forall_{m>k}\left\{J\left(w_{m}, w_{m+1}\right)=0=\gamma(1)-\gamma(1)=\gamma\left(w_{m}\right)-\gamma\left(w_{m+1}\right)\right\}$.

Case 3. If $x=1$, then there exists only one dynamic process $\left(w_{m}: m \in\{0\} \cup \mathbb{N}\right)$ starting at $w_{0}=1$. This dynamic process is of the form $\forall_{m \in \mathbb{N}}\left\{w_{m}=1=T\left(w_{m-1}\right)=T^{[m]}\left(w_{0}\right)\right\}$ and satisfies $\forall_{m \in\{0\} \cup \mathbb{N}}\left\{J\left(w_{m}, w_{m+1}\right)=0 \leqslant \gamma\left(w_{m}\right)-\gamma\left(w_{m+1}\right)\right\}$.

From the above, it follows that, for each $x \in X$, each sequence $\left(w_{m}: m \in\{0\} \cup \mathbb{N}\right)$ starting at $w_{0}=x$ and satisfying (1.1) satisfies (3.4). Thus, the assumption of Theorem $4.4\left(\mathrm{~B}_{1}\right)$ is satisfied.

From the above, it follows also that, for each $x \in X$, each dynamic process $\left(w_{m}: m \in\right.$ $\{0\} \cup \mathbb{N}) \in \mathcal{W}_{2}(T, x)$ starting at $w_{0}=x$ of the system $(X, T)$ converges to $w \in W=\{1\}$ if $x \in\{0,1\}$, to $w \in W=\{0\}$ if $x \in(0,1)$ and $\left(w_{m}: m \in\{0\} \cup \mathbb{N}\right)$ satisfies (5.21), to $w \in W=\{1\}$ if $x \in(0,1)$ and $\left(w_{m}: m \in\{0\} \cup \mathbb{N}\right)$ satisfies (5.22).

This gives that the Theorem $4.4\left(\mathrm{~B}_{1}\right)$ holds.

(b) For each $q \in \mathbb{N}$, the map $T^{[q]}$ is not closed in $X$. 
Indeed, we have that, for each $q \geqslant 2$,

$$
T^{[q]}(x)= \begin{cases}\{1\} & \text { if } x=0 \\ {\left[0,\left(1 / 2^{q}\right) x\right] \cup\{1\}} & \text { if } x \in(0,1) \\ \{1\} & \text { if } x=1 .\end{cases}
$$

Thus, if $q \in N$ and $x \in(0,1)$ are arbitrary and fixed, then $\left[0,\left(1 / 2^{q}\right) x\right] \cup\{1\}=T^{[q]}(x)$ if $q \geqslant 2$ and $[0,(1 / 2) x]=T(x)$. Define the sequence $\left(w_{m}: m \in \mathbb{N}\right)$ as follows: $w_{m}=\left(1 / 2^{q+m}\right) x, m \in \mathbb{N}$. Let $\left(v_{m}: m \in \mathbb{N}\right)$ be a sequence satisfying $\forall_{m \in \mathbb{N}}\left\{v_{m} \in T^{[q]}\left(w_{m}\right)\right\}$ of the form $v_{m}=0 \in T^{[q]}\left(w_{m}\right)$, $m \in \mathbb{N}$. It is clear that $\left(w_{m}: m \in \mathbb{N}\right)$ converges to $w \in W=\{0\},\left(v_{m}: m \in \mathbb{N}\right)$ converges to $v \in V=\{0\}$, and $0 \notin T^{[q]}(0)=\{1\}$.

By Definition 4.1, for each $q \in \mathbb{N}$, the map $T^{[q]}$ is not closed in $X$

(c) The assertion in $\left(B_{2}\right)$ of Theorem 4.4 does not hold.

Indeed, let $x \in(0,1)$ be arbitrary and fixed, and let a dynamic process $\left(w_{m}: m \in\{0\} \cup\right.$ $\mathbb{N}) \in \mathcal{W}_{2}(T, x)$ starting at $w_{0}=x$ of the system $(X, T)$ such that $\forall_{m \in \mathbb{N}}\left\{w_{m} \in(0,1)\right\}$ be arbitrary and fixed. By $(\mathrm{a}),\left(w_{m}: m \in\{0\} \cup \mathbb{N}\right)$ converges to $w \in W=\{0\}$. Since $\forall_{q \in \mathbb{N}}\left\{0 \notin T^{[q]}(0)=\{1\}\right\}$, we see that the assertion in $\left(B_{2}\right)$ of Theorem 4.4 does not hold.

It is worth noticing that if $x \in\{0,1\}$ and $\left(w_{m}: m \in\{0\} \cup \mathbb{N}\right) \in \mathcal{W}_{2}(T, x)$, then, by (a), $\left(w_{m}: m \in\{0\} \cup \mathbb{N}\right)$ converges to $w \in W=\{1\}$ and $\forall_{q \in \mathbb{N}}\left\{1 \in T^{[q]}(1)=\{1\}\right\}$, that is, $\forall_{q \in \mathbb{N}}\left\{1 \in \operatorname{End}\left(T^{[q]}\right)\right\}$.

(d) The map $T$ is not strictly $(2, \Gamma)$ dissipative on $X$.

Indeed, if $x \in(0,1)$ is arbitrary and fixed, then we have that $\forall_{m \in \mathbb{N}}\left\{T^{[m]}\left(w_{0}\right)=\right.$ $\left.\left[0,\left(1 / 2^{m}\right) w_{0}\right] \cup\{1\}\right\}$ for $w_{0}=x$ and the generalized sequence of iterations $\left(w_{m}: m \in\{0\} \cup \mathbb{N}\right)$ defined by $w_{1}=(1 / 2) w_{0} \in T\left(w_{0}\right)$ and $\forall_{m \geqslant 2}\left\{w_{m}=0 \in T^{[m]}\left(w_{0}\right)\right\}$ does not satisfy (3.4) since $0 \leqslant J\left(w_{1}, w_{2}\right)=\left|w_{1}-w_{2}\right|=(1 / 2) w_{0}>\gamma\left(w_{1}\right)-\gamma\left(w_{2}\right)=(1 / 2) w_{0}-1$.

In Example 5.5, we compare Theorem 4.4 and [2].

Example 5.5. Let $X, T$, and $p$ be as in Example 5.3.,

We show that $T$, is not a generalized contraction of Reilly [2]. Indeed, suppose that $\exists_{\lambda \in[0,1)} \forall_{x, y \in X}\{p(T(x), T(y)) \leqslant \lambda p(x, y)\}$. Hence, in particular, for $x_{0}=5$, and $y_{0}=6$, we get $T\left(x_{0}\right)=3, T\left(y_{0}\right)=5$ and $p\left(T\left(x_{0}\right), T\left(y_{0}\right)\right)=1 \leqslant \lambda \cdot 1=\lambda p\left(x_{0}, y_{0}\right)$. This gives $\lambda \geqslant 1$, which is absurd.

\section{References}

[1] I. L. Reilly, “Quasi-gauge spaces," Journal of the London Mathematical Society, vol. 6, pp. 481-487, 1973.

[2] I. L. Reilly, "A generalized contraction principle," Bulletin of the Australian Mathematical Society, vol. 10, pp. 359-363, 1974.

[3] I. L. Reilly, P. V. Subrahmanyam, and M. K. Vamanamurthy, "Cauchy sequences in quasipseudometric spaces," Monatshefte für Mathematik, vol. 93, no. 2, pp. 127-140, 1982.

[4] J. C. Kelly, "Bitopological spaces," Proceedings of the London Mathematical Society, vol. 13, pp. 71-89, 1963.

[5] P. V. Subrahmanyam, "Remarks on some fixed-point theorems related to Banach's contraction principle," Journal of Mathematical and Physical Sciences, vol. 8, pp. 445-457, 1974.

[6] E. Alemany and S. Romaguera, "On right $K$-sequentially complete quasi-metric spaces," Acta Mathematica Hungarica, vol. 75, no. 3, pp. 267-278, 1997. 
[7] S. Romaguera, "Left K-completeness in quasi-metric spaces," Mathematische Nachrichten, vol. 157, pp. 15-23, 1992.

[8] R. A. Stoltenberg, “On quasi-metric spaces,” Duke Mathematical Journal, vol. 36, pp. 65-71, 1969.

[9] W. A. Wilson, "On quasi-metric spaces," American Journal of Mathematics, vol. 53, no. 3, pp. 675-684, 1931.

[10] V. Gregori and S. Romaguera, "Fixed point theorems for fuzzy mappings in quasi-metric spaces," Fuzzy Sets and Systems, vol. 115, no. 3, pp. 477-483, 2000.

[11] G. M. Lee, B. S. Lee, J. S. Jung, and S. Chang, "Minimization theorems and fixed point theorems in generating spaces of quasi-metric family," Fuzzy Sets and Systems, vol. 101, no. 1, pp. 143-152, 1999.

[12] M. Frigon, "Fixed point results for generalized contractions in gauge spaces and applications," Proceedings of the American Mathematical Society, vol. 128, no. 10, pp. 2957-2965, 2000.

[13] A. Chiş-Novac, R. Precup, and I. A. Rus, "Data dependence of fixed points for non-self generalized contractions," Fixed Point Theory, vol. 10, no. 1, pp. 73-87, 2009.

[14] P. Fletcher and W. F. Lindgren, Quasi-Uniform Spaces, vol. 77 of Lecture Notes in Pure and Applied Mathematics, Marcel Dekker, New York, NY, USA, 1982.

[15] H.-P. A. Künzi, “Quasi-uniform spaces—eleven years later,” Topology Proceedings, vol. 18, pp. 143-171, 1993.

[16] H.-P. A. Künzi, "Nonsymmetric topology," in Topology with Applications, vol. 4 of Bolyai Society Mathematical Studies, pp. 303-338, János Bolyai Mathematical Society, Budapest, Hungary, 1995.

[17] J.-P. Aubin and J. Siegel, "Fixed points and stationary points of dissipative multivalued maps," Proceedings of the American Mathematical Society, vol. 78, no. 3, pp. 391-398, 1980.

[18] J.-P. Aubin and I. Ekeland, Applied Nonlinear Analysis, Pure and Applied Mathematics, John Wiley \& Sons, New York, NY, USA, 1984.

[19] J.-P. Aubin and H. Frankowska, Set-Valued Analysis, vol. 2 of Systems $\mathcal{E}$ Control: Foundations $\mathcal{E}$ Applications, Birkhäuser, Boston, Mass, USA, 1990.

[20] G. X.-Z. Yuan, KKM Theory and Applications in Nonlinear Analysis, vol. 218 of Monographs and Textbooks in Pure and Applied Mathematics, Marcel Dekker, New York, NY, USA, 1999.

[21] E. Tarafdar and R. Vyborny, A Generalized (Multivalued) Contraction Mapping Principle, vol. 54 of Research Reports in Pure Mathematics, The University of Queensland, Queensland, Australia, 1976.

[22] E. Tarafdar and X.-Z. Yuan, "Set-valued topological contractions," Applied Mathematics Letters, vol. 8, no. 6, pp. 79-81, 1995.

[23] S. Banach, "Sur les opérations dans les ensembles abstraits et leurs applications aux équations intégrales," Fundamenta Mathematicae, vol. 3, pp. 133-181, 1922.

[24] M. Edelstein, "On fixed and periodic points under contractive mappings," Journal of the London Mathematical Society, vol. 37, pp. 74-79, 1962.

[25] J. Caristi, "Fixed point theorems for mappings satisfying inwardness conditions," Transactions of the American Mathematical Society, vol. 215, pp. 241-251, 1976.

[26] J. Caristi and W. A. Kirk, "Geometric fixed point theory and inwardness conditions," in The Geometry of Metric and Linear Spaces, vol. 490 of Lecture Notes in Mathematics, pp. 74-83, Springer, Berlin, Germany, 1975.

[27] W. A. Kirk and L. M. Saliga, "The Brézis-Browder order principle and extensions of Caristi's theorem," Nonlinear Analysis: Theory, Methods \& Applications, vol. 47, no. 4, pp. 2765-2778, 2001.

[28] I. Ekeland, "On the variational principle," Journal of Mathematical Analysis and Applications, vol. 47, pp. 324-353, 1974.

[29] C. Ding and S. B. Nadler Jr., "The periodic points and the invariant set of an $\varepsilon$-contractive map," Applied Mathematics Letters, vol. 15, no. 7, pp. 793-801, 2002.

[30] S. B. Nadler Jr., "Periodic points of multi-valued $\varepsilon$-contractive maps," Topological Methods in Nonlinear Analysis, vol. 22, no. 2, pp. 399-409, 2003.

[31] L. Cirić, "Periodic and fixed point theorems in a quasi-metric space," Australian Mathematical Society Journal, vol. 54, no. 1, pp. 80-85, 1993.

[32] J. C. Willems, "Dissipative dynamical systems. I. General theory," Archive for Rational Mechanics and Analysis, vol. 45, pp. 321-351, 1972.

[33] W. Zangwill, Nonlinear Programming, Prentice-Hall, Englewood Cliffs, NJ, USA, 1969.

[34] M. Justman, "Iterative processes with "nucleolar" restrictions," International Journal of Game Theory, vol. 6, no. 4, pp. 189-212, 1978. 
[35] M. Maschler and B. Peleg, "Stable sets and stable points of set-valued dynamic systems with applications to game theory," SIAM Journal on Control and Optimization, vol. 14, no. 6, pp. 985-995, 1976.

[36] A. Petruşel and A. Sîntămărian, "Single-valued and multi-valued Caristi type operators," Publicationes Mathematicae Debrecen, vol. 60, no. 1-2, pp. 167-177, 2002.

[37] K. Włodarczyk and R. Plebaniak, "Maximality principle and general results of Ekeland and Caristi types without lower semicontinuity assumptions in cone uniform spaces with generalized pseudodistances," Fixed Point Theory and Applications, vol. 2010, Article ID 175453, 35 pages, 2010.

[38] K. Włodarczyk and R. Plebaniak, "Periodic point, endpoint, and convergence theorems for dissipative set-valued dynamic systems with generalized pseudodistances in cone uniform and uniform spaces," Fixed Point Theory and Applications, vol. 2010, Article ID 864536, 32 pages, 2010.

[39] K. Włodarczyk, R. Plebaniak, and M. Doliński, “Cone uniform, cone locally convex and cone metric spaces, endpoints, set-valued dynamic systems and quasi-asymptotic contractions," Nonlinear Analysis: Theory, Methods \& Applications, vol. 71, no. 10, pp. 5022-5031, 2009.

[40] K. Włodarczyk, R. Plebaniak, and C. Obczyński, "Convergence theorems, best approximation and best proximity for set-valued dynamic systems of relatively quasi-asymptotic contractions in cone uniform spaces," Nonlinear Analysis: Theory, Methods E Applications, vol. 72, no. 2, pp. 794-805, 2010.

[41] K. Włodarczyk and R. Plebaniak, "A fixed point theorem of Subrahmanyam type in uniform spaces with generalized pseudodistances," Applied Mathematics Letters, vol. 24, no. 3, pp. 325-328, 2011.

[42] J. Dugundji, Topology, Allyn and Bacon, Boston, Mass, USA, 1966.

[43] C. Berge, Topological Spaces, Oliver \& Boyd, Edinburgh, UK, 1963.

[44] E. Klein and A. C. Thompson, Theory of Correspondences: Including Applications to Mathematical Economics, Canadian Mathematical Society Series of Monographs and Advanced Texts, John Wiley \& Sons, New York, NY, USA, 1984. 